\title{
Association between polymorphisms in microRNAs and ischemic stroke in an Asian population: evidence based on 6,083 cases and 7,248 controls
}

This article was published in the following Dove Press journal:

Clinical Interventions in Aging

\section{Donghua Zou ${ }^{1, *}$ \\ Chunbin Liu',** \\ Qian Zhang' \\ Xianfeng $\mathrm{Li}^{\prime}$ \\ Gang Qin' \\ Qi Huang' \\ Youshi Meng' \\ Li Chen² \\ Jinru Wei'}

'Department of Stroke Center,

The Fifth Affiliated Hospital of

Guangxi Medical University, Nanning,

Guangxi, People's Republic of China;

${ }^{2}$ Department of Neurology, The First

Affiliated Hospital of Guangxi Medical

University, Nanning, Guangxi, People's

Republic of China

*These authors contributed equally to this work

Correspondence: Jinru Wei

Department of Stroke Center, The

Fifth Affiliated Hospital of Guangxi

Medical University, No 89 Qixing Road,

Nanning, Guangxi 530022, People's

Republic of China

Tel +86 77I 26I 7892

Email drweijinru@।26.com

Li Chen

Department of Neurology, The First Affiliated Hospital of Guangxi Medical University, No 6 Shuangyong

Road, Nanning, Guangxi 53002I,

People's Republic of China

Tel +86 77l 535003 |

Email chenliqfkk@I63.com

\begin{abstract}
Background: Polymorphisms in miR-146a (rs2910164), miR-196a2 (rs11614913), miR-149 (rs2292832) and miR-499 (rs3746444) have been associated with ischemic stroke (IS), but studies have given inconsistent results.

Methods: This meta-analysis investigated the possible association between IS risk and the four polymorphisms. A total of 14 case-control studies from Asian populations involving 6,083 cases and 7,248 controls for the four polymorphisms were included.

Results: Results showed that the GG genotype of miR-146a (rs2910164) may be associated with increased IS risk according to the recessive model $(\mathrm{OR}=1.20,95 \% \mathrm{CI}=1.02-1.42, P=0.03)$. Similarly, the CC genotype of miR-149 (rs2292832) may be associated with increased IS risk according to the recessive model $(\mathrm{OR}=1.28,95 \% \mathrm{CI}=1.08-1.52, P=0.005)$ and the homozygous model $(\mathrm{OR}=1.31,95 \% \mathrm{CI}=1.09-1.58, P=0.004)$. In contrast, miR-196a2 (rs11614913) and miR-499 (rs3746444) polymorphisms did not show significant association with IS risk in any of the five genetic models.
\end{abstract}

Conclusion: These results indicate that the GG genotype of miR-146a (rs2910164) and CC genotype of miR-149 (rs2292832) may confer increased susceptibility to IS, while miR-196a2 (rs11614913) and miR-499 (rs3746444) polymorphisms may not be associated with IS risk in Asian populations. These conclusions should be verified in large and well-designed studies.

Keywords: miRNAs, polymorphism, ischemic stroke, meta-analysis

\section{Introduction}

Stroke is a significant worldwide problem. An estimated $80 \%$ of the patients survive for at least 1 year after stroke, yet $>70 \%$ have enduring disabilities. ${ }^{1,2}$ Ischemic stroke (IS) and intracerebral hemorrhage account for $\sim 80 \%-85 \%$ and $15 \%-20 \%$ of all stroke cases, respectively. ${ }^{3}$ IS is a complex syndrome whose pathological development involves multiple components, which include environmental and genetic factors. ${ }^{4}$ Established environmental risk factors include age, sex, body mass index, hypertension, diabetes mellitus, smoking, and hyperlipidemia. However, recent studies suggested that genetics may contribute more than environment to IS, considering that a number of single-gene disorders are related to IS. ${ }^{5-8}$ Nevertheless, the factors defining genetic susceptibility to IS remain unclear.

MicroRNAs (miRNAs) represent a group of short non-coding RNA molecules, 18-25 nucleotides in length. Bioinformatics data indicate that a single miRNA can bind to as many as 200 gene targets, and miRNAs may regulate the expression of approximately one-third of protein-coding mRNAs. A single-nucleotide polymorphism 
(SNP) in miRNA may create a mismatch, leading to gene expression disorder and diseases. ${ }^{9}$ Evidence has indicated that miRNAs regulate various IS-related biological processes, such as atherosclerosis, hypertension, and plaque rupture. ${ }^{10}$ In fact, altered miRNA expression has been observed in IS in preclinical animal models and patients, suggesting a potential role in predicting the diagnosis and prognosis of IS. ${ }^{11,12}$

More specifically, the literature suggests an association between IS and polymorphisms in miR-146a (rs2910164), miR-196a2 (rs11614913), miR-149 (rs2292832), and miR-499 (rs3746444). ${ }^{13-26}$ However, these associations are controversial because individual studies relied on relatively small samples. Therefore, to obtain a more comprehensive understanding of the available evidence, we conducted this meta-analysis of 14 case-control studies to evaluate the possible association between IS risk and miR-146a (rs2910164), miR-196a2 (rs11614913), miR-149 (rs2292832), and miR-499 (rs3746444) in Asian populations.

\section{Materials and methods}

\section{Search strategy}

All clinical and experimental case-control studies of miRNA polymorphisms and IS risk published through February 1, 2018 were identified through systematic searches in PubMed, EMBASE, Google Scholar, and the Chinese National Knowledge Infrastructure (CNKI) databases using English and Chinese. The search terms used were as follows: microRNA; miRNA; these two terms in combination with polymorphism, polymorphisms, SNP, variant, variants, variation, genotype, genetic, or mutation; and all the abovementioned terms in combination with stroke or ischemic stroke. Reference lists in identified articles and reviews were also searched manually to identify additional eligible studies.

\section{Inclusion criteria}

To be included in our review and meta-analysis, studies had to 1) have a case-control design for assessing the association of IS risk with miR-146a (rs2910164), miR196a2 (rs11614913), miR-149 (rs2292832), and miR-499 (rs3746444); 2) be accessible as a full-text article and report sufficient data for estimating ORs with 95\% CIs; 3) report genotype frequencies; and 4) involve humans rather than animal models.

\section{Data extraction}

Two authors (DHZ and CBL) independently extracted the following data from the included studies: first author's family name, year of publication, ethnicity, testing methods, control source, age, sex, $P$-value for Hardy-Weinberg equilibrium (HWE) in controls, numbers and genotypes of cases and controls, and frequencies of genotypes in cases and controls. Discrepancies were resolved by consensus. Only those studies that met the predetermined inclusion criteria were included.

\section{Assessment of methodological quality}

To assess the quality of the studies included in this analysis, the Newcastle-Ottawa scale was used by two independent assessors (JRW and LC). ${ }^{27}$ For the Newcastle-Ottawa scale, a full score is nine stars; a score range of 5-9 stars is considered to indicate generally high methodological quality, whereas a range of $0-4$ stars is considered to indicate poor quality. ${ }^{28}$ The quality of all the included studies is summarized in Table 1. Any disagreements about Newcastle-Ottawa scores were resolved by other authors following a comprehensive reassessment. Only high-quality studies were included in our meta-analysis.

\section{Statistical analyses}

The unadjusted OR with 95\% CI was used to assess the strength of the association of IS risk with miR146a (rs2910164), miR-196a2 (rs11614913), miR-149 (rs2292832), and miR-499 (rs3746444) based on genotype frequencies in cases and controls. The significance of pooled ORs was determined using the $Z$-test, with $P<0.05$ defined as the significance threshold. Meta-analysis was conducted using a fixed-effect model when $P>0.10$ for the $Q$-test, indicating the lack of heterogeneity among studies; otherwise, a random-effect model was used. All these statistical tests were performed using Review Manager 5.2 (Cochrane Collaboration, Oxford, England).

Publication bias was assessed using Begg's funnel plot and Egger's weighted regression, with $P<0.05$ considered statistically significant. Begg's funnel plots and Egger's weighted regression were calculated using Stata 12.0 (StataCorp LP, College Station, TX, USA).

\section{Results \\ Description of studies}

Figure 1 is a flow diagram illustrating the process of searching for and selecting studies. A total of 184 potentially relevant publications up to February 1, 2018 were systematically identified through searches of the PubMed, EMBASE, Google Scholar, and CNKI databases in English 


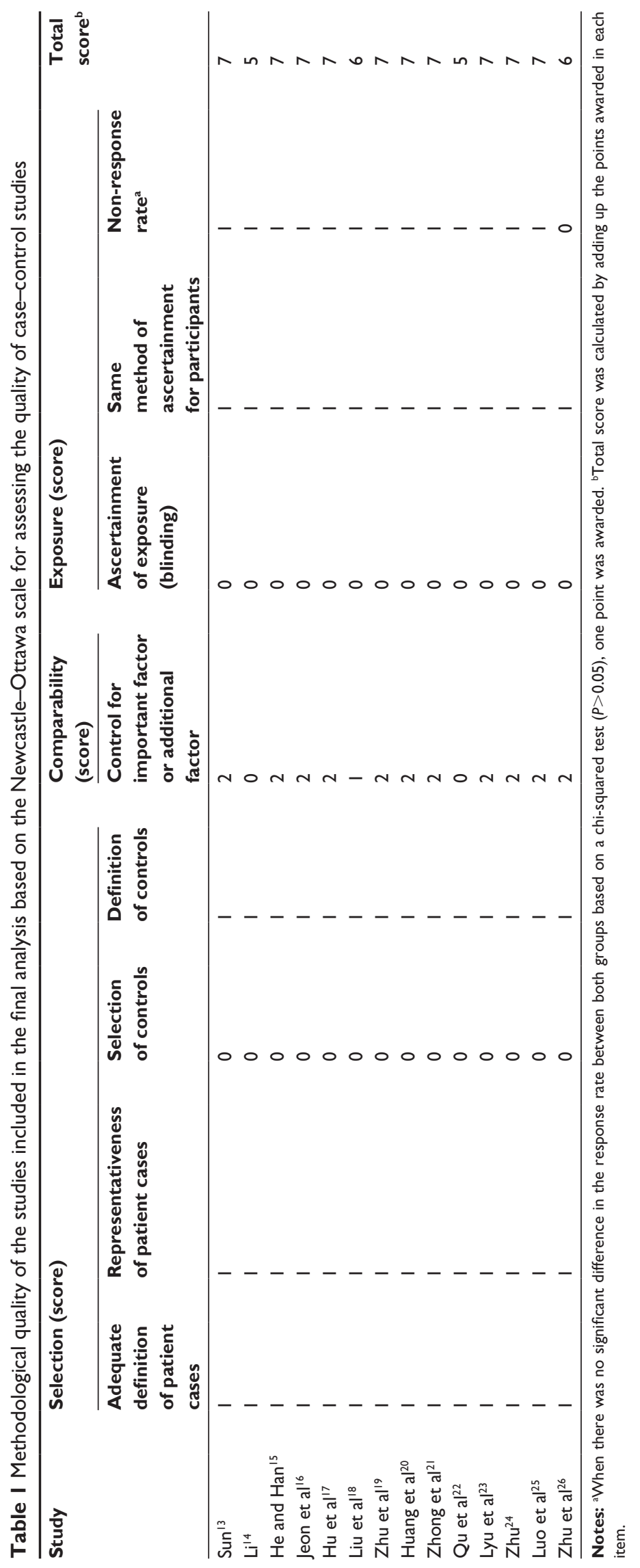


184 potentially relevant studies identified through PubMed, EMBASE, Google Scholar, and the Chinese National Knowledge Infrastructure databases using English and Chinese up to February 1, 2018

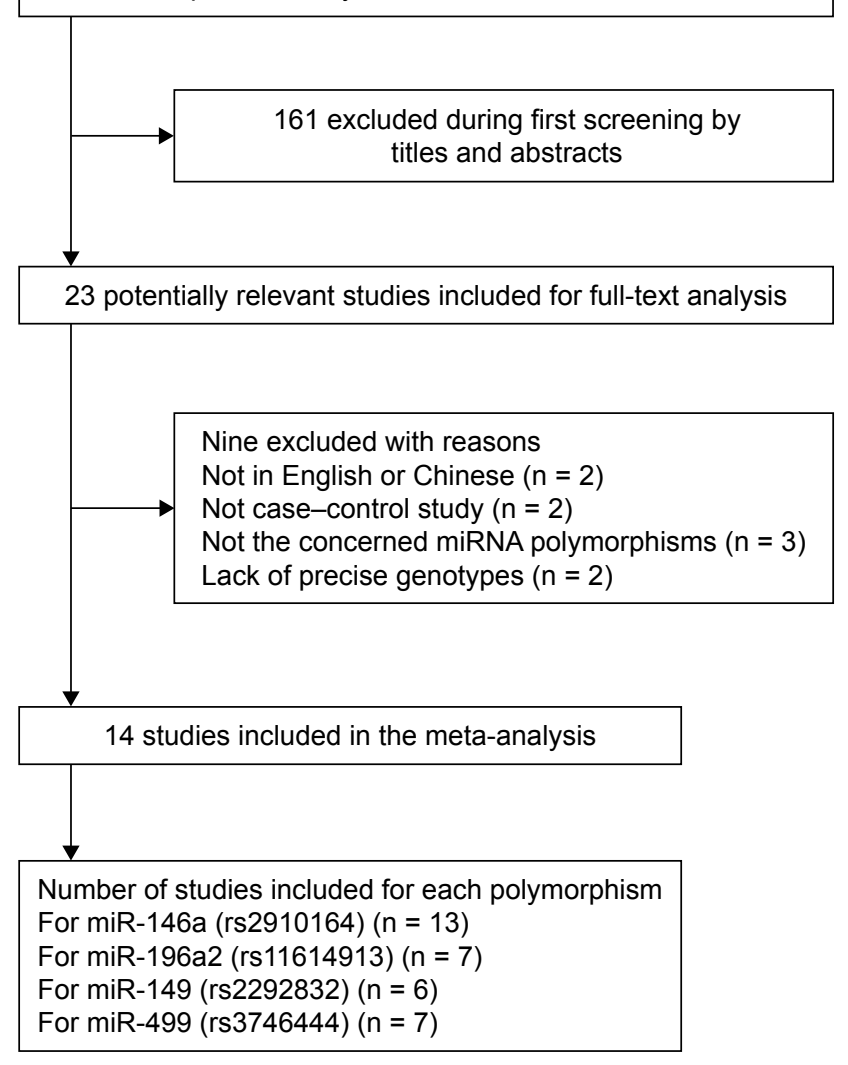

Figure I Flowchart of study selection.

and Chinese. Of these, we excluded 161 studies during initial screening based on review of the titles and abstracts. During analysis of the full text of the remaining articles, two studies were excluded for not being case-control studies, three studies were excluded because they did not report precise genotypes, and two articles were excluded because they investigated polymorphisms of miRNAs other than miR-146a (rs2910164), miR-196a2 (rs11614913), miR-149 (rs2292832), or miR-499 (rs3746444). A further two studies were excluded because they were not written in English or Chinese.

In the end, 14 studies $^{13-26}$ were included in this metaanalysis based on our search strategy and inclusion criteria. Their characteristics are summarized in Table 2. Of these, 13 studies $^{13,14,16-26}$ (Table 3) involving 5,726 cases and 7,175 controls evaluated the association between miR-146a (rs2910164) polymorphism and IS risk. Seven studies ${ }^{16,18-20,}$ 24-26 (Table 3) involving 3,090 cases and 3,047 controls evaluated the association between miR-196a2 (rs11614913) polymorphism and IS risk. Six studies ${ }^{15-17,24-26}$ (Table 3) involving 2,448 cases and 2,322 controls evaluated miR-149 (rs2292832) polymorphism and IS risk. The remaining seven studies $^{16,18,20,23-26}$ (Table 3) involving 3,082 cases and 3,044 controls evaluated miR-499 (rs3746444) polymorphism and IS risk. The distribution of genotypes in controls was consistent with HWE $(P>0.05)$ in all but three studies. ${ }^{14,20,22}$ The overall quality of the included studies was adequate, and the mean Newcastle-Ottawa score for the included studies was 6.57 (Table 1).

\section{Quantitative data synthesis}

IS risk and miR-I46a (rs29l0164) polymorphism

The overall results for miR-146a (rs2910164) are summarized in Table 4 and Figure 2. On the basis of 5,726 cases and 7,175 controls from 13 studies, ${ }^{13,14,16-26}$ the overall results indicated that the GG genotype of miR-146a (rs2910164) may be associated with increased IS risk according to the recessive model $(\mathrm{OR}=1.20,95 \% \mathrm{CI}=1.02-1.42, P=0.03$; Figure 2B).

Table 2 Characteristics of the studies included in the meta-analysis

\begin{tabular}{|c|c|c|c|c|c|c|c|c|c|c|}
\hline \multirow[t]{2}{*}{ Study } & \multirow[t]{2}{*}{ Year } & \multirow[t]{2}{*}{ Ethnicity } & \multirow[t]{2}{*}{ Country } & \multirow{2}{*}{$\begin{array}{l}\text { Testing } \\
\text { method }\end{array}$} & \multirow{2}{*}{$\begin{array}{l}\text { Control } \\
\text { source }\end{array}$} & \multicolumn{2}{|c|}{ Age (years, mean $\pm S D$ ) } & \multicolumn{2}{|c|}{ Male, n (\%) } & \multirow[t]{2}{*}{ SNP } \\
\hline & & & & & & Cases & Controls & Cases & Controls & \\
\hline Sun ${ }^{13}$ & 2011 & Asian & China & PCR-RFLP & $\begin{array}{l}\text { Hospital- } \\
\text { based healthy } \\
\text { volunteers }\end{array}$ & $63 \pm 12$ & $62 \pm 13$ & $236(61.9)$ & $347(53.4)$ & $\begin{array}{l}\text { miR-I46a } \\
(\mathrm{rs} 2910164)\end{array}$ \\
\hline $\mathrm{Li}^{14}$ & 2010 & Asian & China & PCR-RFLP & $\begin{array}{l}\text { Hospital- } \\
\text { based healthy } \\
\text { volunteers }\end{array}$ & $64 \pm 11$ & $45 \pm 12$ & $188(67.2)$ & $579(57.3)$ & $\begin{array}{l}\text { miR-I46a } \\
(\mathrm{rs} 2910164)\end{array}$ \\
\hline $\mathrm{He}$ and $\mathrm{Han}^{15}$ & 2013 & Asian & China & PCR-RFLP & $\begin{array}{l}\text { Hospital- } \\
\text { based healthy } \\
\text { volunteers }\end{array}$ & $65.7 \pm 11.5$ & $66.3 \pm 10.2$ & $205(55.0)$ & $193(51.7)$ & $\begin{array}{l}\text { miR-I49 } \\
\text { (rs2292832) }\end{array}$ \\
\hline Jeon et $\mathrm{al}^{16}$ & 2013 & Asian & $\begin{array}{l}\text { South } \\
\text { Korea }\end{array}$ & TaqMan & $\begin{array}{l}\text { Hospital- } \\
\text { based healthy } \\
\text { volunteers }\end{array}$ & $64.16 \pm 11.90$ & $63.14 \pm 10.19$ & $336(49.6)$ & $244(44.1)$ & $\begin{array}{l}\text { miR-I46a } \\
\text { (rs29l0I64); } \\
\text { miR-196a2 } \\
\text { (rs|l6|49|3); }\end{array}$ \\
\hline
\end{tabular}


Table 2 (Continued)

\begin{tabular}{|c|c|c|c|c|c|c|c|c|c|c|}
\hline \multirow[t]{2}{*}{ Study } & \multirow[t]{2}{*}{ Year } & \multirow[t]{2}{*}{ Ethnicity } & \multirow[t]{2}{*}{ Country } & \multirow{2}{*}{$\begin{array}{l}\text { Testing } \\
\text { method }\end{array}$} & \multirow{2}{*}{$\begin{array}{l}\text { Control } \\
\text { source }\end{array}$} & \multicolumn{2}{|c|}{ Age (years, mean \pm SD) } & \multicolumn{2}{|l|}{ Male, $\mathbf{n}(\%)$} & \multirow[t]{2}{*}{ SNP } \\
\hline & & & & & & Cases & Controls & Cases & Controls & \\
\hline & & & & & & & & & & miR-I49 \\
\hline & & & & & & & & & & (rs2292832); \\
\hline & & & & & & & & & & and miR-499 \\
\hline & & & & & & & & & & (rs3746444) \\
\hline \multirow[t]{3}{*}{ Hu et $\mathrm{a}^{17}$} & 2014 & Asian & China & PCR-RFLP & Hospital- & $64 \pm 11.7$ & $63 \pm 10.5$ & $94(48.0)$ & $95(46.3)$ & miR-I46a \\
\hline & & & & & based healthy & & & & & (rs2910164) \\
\hline & & & & & volunteers & & & & & and miR-I49 \\
\hline & & & & & & & & & & (rs2292832) \\
\hline \multirow[t]{6}{*}{ Liu et $\mathrm{al}^{18}$} & 2014 & Asian & China & PCR-RFLP & Hospital- & $67.52 \pm 10.29$ & $66.34 \pm 11.07$ & $227(58.06)$ & $180(60.81)$ & miR-I46a \\
\hline & & & & & based healthy & & & & & (rs2910164); \\
\hline & & & & & volunteers & & & & & miR-196a2 \\
\hline & & & & & & & & & & (rs|l6|49|3); \\
\hline & & & & & & & & & & and miR-499 \\
\hline & & & & & & & & & & $(\mathrm{rs} 3746444)$ \\
\hline \multirow[t]{3}{*}{ Zhu et al ${ }^{19}$} & 2014 & Asian & China & PCR-LDR & Hospital- & $61.62 \pm 0.986$ & $62.05 \pm 0.982$ & $253(68.75)$ & $261(68.50)$ & miR-I46a \\
\hline & & & & & based healthy & & & & & (rs2910164) \\
\hline & & & & & volunteers & & & & & and $\mathrm{miR}-196 \mathrm{a} 2$ \\
\hline \multirow{7}{*}{ Huang et $\mathrm{al}^{20}$} & & & & & & & & & & $(r s \mid 1614913)$ \\
\hline & 2015 & Asian & China & TaqMan & Hospital- & $63(54-70)^{\mathrm{a}}$ & $61(54-68)^{a}$ & $327(61.6)$ & $327(6 \mid .6)$ & miR-I46a \\
\hline & & & & & based healthy & & & & & (rs2910164); \\
\hline & & & & & volunteers & & & & & miR-196a2 \\
\hline & & & & & & & & & & (rs|l6|49|3); \\
\hline & & & & & & & & & & and miR-499 \\
\hline & & & & & & & & & & (rs3746444) \\
\hline \multirow[t]{3}{*}{ Zhong et $\mathrm{al}^{21}$} & 2016 & Asian & China & PCR & Hospital- & $62.6 \pm 8.63$ & $61.1 \pm 9.58$ & 177 (59.6) & $170(56.7)$ & miR-I46a \\
\hline & & & & & based healthy & & & & & (rs2910164) \\
\hline & & & & & volunteers & & & & & \\
\hline Qu et $\mathrm{al}^{22}$ & 2016 & Asian & China & PCR-LDR & Hospital- & $61.30 \pm 9.40$ & $59.50 \pm 8.50$ & $718(63.0)$ & $903(57.0)$ & miR-I46a \\
\hline & & & & & based healthy & & & & & $(\mathrm{rs} 2910164)$ \\
\hline & & & & & volunteers & & & & & \\
\hline Lyu et $\mathrm{al}^{23}$ & 2016 & Asian & China & TaqMan & Hospital- & $58 \pm 11.9$ & $58 \pm 11.9$ & $210(55.6)$ & $210(55.6)$ & miR-I46a \\
\hline & & & & & based healthy & & & & & (rs2910164) \\
\hline & & & & & volunteers & & & & & and miR-499 \\
\hline & & & & & & & & & & (rs3746444) \\
\hline Zhu ${ }^{24}$ & 2016 & Asian & China & PCR-RFLP & Hospital- & $63.74 \pm 4.49$ & $63.31 \pm 4.84$ & $215(54.3)$ & $202(53.4)$ & miR-146a \\
\hline & & & & & based healthy & & & & & (rs2910164); \\
\hline & & & & & volunteers & & & & & miR-196a2 \\
\hline & & & & & & & & & & (rsII6I49|3); \\
\hline & & & & & & & & & & miR-I49 \\
\hline & & & & & & & & & & (rs2292832); \\
\hline & & & & & & & & & & and miR-499 \\
\hline & & & & & & & & & & (rs3746444) \\
\hline Luo et $\mathrm{al}^{25}$ & 2017 & Asian & China & PCR & Hospital- & $67.70 \pm 12.33$ & $60.17 \pm 10.32$ & $196(65.8)$ & |8| (59.8) & miR-I $46 a$ \\
\hline & & & & & based healthy & & & & & (rs2910164); \\
\hline & & & & & volunteers & & & & & miR-196a2 \\
\hline & & & & & & & & & & (rs|l6I49|3); \\
\hline & & & & & & & & & & miR-I49 \\
\hline & & & & & & & & & & (rs2292832); \\
\hline & & & & & & & & & & and miR-499 \\
\hline & & & & & & & & & & (rs3746444) \\
\hline Zhu et $\mathrm{al}^{26}$ & 2017 & Asian & China & TaqMan & Hospital- & $61.0 \pm 10.2$ & $59.7 \pm 9.9$ & $321(62.9)$ & $311(59.4)$ & miR-I46a \\
\hline & & & & & based healthy & & & & & (rs29l0164); \\
\hline & & & & & volunteers & & & & & miR-196a2 \\
\hline & & & & & & & & & & (rs|l6|49|3); \\
\hline & & & & & & & & & & miR-I49 \\
\hline & & & & & & & & & & (rs2292832); \\
\hline & & & & & & & & & & and miR-499 \\
\hline & & & & & & & & & & (rs3746444) \\
\hline
\end{tabular}

Note: ${ }^{2}$ These data are expressed as median (25th, 75 th quartiles).

Abbreviations: LDR, ligase detection reaction; PCR, polymerase chain reaction; RFLP, restriction fragment length polymorphism; SNP, single-nucleotide polymorphism. 
Table 3 Genotype distributions of miR-I46a (rs29I0I64), miR-196a2 (rs I I6I49I3), miR-I49 (rs2292832), and miR-499 (rs3746444)

\begin{tabular}{|c|c|c|c|c|c|c|c|c|c|c|c|c|c|}
\hline \multirow{2}{*}{\multicolumn{2}{|c|}{$\begin{array}{l}\text { Study Year } \\
\text { miR-I 46a (rs29 } 10164\end{array}$}} & \multirow{2}{*}{$\begin{array}{l}\text { P-value } \\
\text { for } \\
\text { HWE } \\
\text { ) }\end{array}$} & \multirow[t]{2}{*}{$\begin{array}{l}\text { Sample size } \\
\text { (cases/controls) }\end{array}$} & \multicolumn{3}{|c|}{$\begin{array}{l}\text { No of } \\
\text { cases }\end{array}$} & \multicolumn{2}{|c|}{$\begin{array}{l}\text { Allele frequencies } \\
\text { of cases, } n(\%)\end{array}$} & \multicolumn{3}{|c|}{$\begin{array}{l}\text { No of } \\
\text { controls }\end{array}$} & \multicolumn{2}{|c|}{$\begin{array}{l}\text { Allele frequencies of } \\
\text { controls, } n(\%)\end{array}$} \\
\hline & & & & \multirow{2}{*}{$\begin{array}{l}\text { CC } \\
136\end{array}$} & \multirow{2}{*}{$\frac{\text { GC }}{161}$} & \multirow{2}{*}{$\begin{array}{l}\mathbf{G G} \\
6 I\end{array}$} & \multirow{2}{*}{$\begin{array}{l}\text { C } \\
433(60.5)\end{array}$} & \multirow{2}{*}{$\begin{array}{l}\text { G } \\
283(39.5)\end{array}$} & \multirow{2}{*}{$\begin{array}{l}\mathbf{C C} \\
228\end{array}$} & \multirow{2}{*}{$\begin{array}{l}\text { GC } \\
304\end{array}$} & \multirow{2}{*}{$\begin{array}{l}\mathbf{G G} \\
118\end{array}$} & \multirow{2}{*}{$\begin{array}{l}\mathbf{C} \\
760(58.5)\end{array}$} & \multirow{2}{*}{$\begin{array}{l}\text { G } \\
540(4 I .5)\end{array}$} \\
\hline $\operatorname{Sun}^{13}$ & 2011 & 0.345 & $358 / 650$ & & & & & & & & & & \\
\hline $\mathrm{Li}^{14}$ & 2010 & 0.009 & $268 / 1,010$ & 79 & 110 & 79 & $268(50.0)$ & $268(50.0)$ & 345 & 455 & 210 & $\mathrm{I}, \mathrm{I} 45$ (56.7) & $875(43.3)$ \\
\hline Jeon et $\mathrm{al}^{16}$ & 2013 & 0.589 & $678 / 553$ & 223 & 327 & 128 & $773(57.0)$ & $583(43.0)$ & 211 & 266 & 76 & $688(62.2)$ & 418 (37.8) \\
\hline Hu et al ${ }^{17}$ & 2014 & 0.193 & $196 / 205$ & 75 & 87 & 34 & $237(60.5)$ & 155 (39.5) & 97 & 82 & 26 & $276(67.3)$ & 134 (32.7) \\
\hline Liu et $\mathrm{a}^{18}$ & 2014 & 0.650 & $296 / 391$ & 85 & 159 & 52 & $329(55.6)$ & $263(44.4)$ & 116 & 198 & 77 & $430(55.0)$ & $352(45.0)$ \\
\hline Zhu et $\mathrm{al}^{19}$ & 2014 & 0.952 & $368 / 381$ & 145 & 173 & 50 & $463(63.0)$ & $273(37.0)$ & 132 & 185 & 64 & $449(80.6)$ & $313(19.4)$ \\
\hline Huang et $\mathrm{a}^{20}$ & 2015 & 0.106 & $53|/ 53|$ & 189 & 261 & 81 & $639(60.2)$ & $423(39.8)$ & 219 & 257 & 55 & $695(65.4)$ & $367(34.6)$ \\
\hline Zhong et $\mathrm{al}^{21}$ & 2016 & 0.133 & $297 / 300$ & $|4|$ & 128 & 28 & $410(69.0)$ & $184(3 \mid .0)$ & 113 & 152 & 35 & $378(63.0)$ & $222(37.0)$ \\
\hline Qu et $a^{22}$ & 2016 & $<0.001$ & I, I39/I,585 & 355 & 618 & 166 & $\mathrm{I}, 328(58.3)$ & $950(41.7)$ & 483 & 869 & 233 & I,835 (57.9) & I,335 (42.I) \\
\hline Lyu et $\mathrm{a}^{23}$ & 2016 & 0.079 & $378 / 378$ & 119 & 198 & 61 & $436(57.7)$ & $320(42.3)$ & 153 & 187 & 38 & $493(65.2)$ & $263(34.8)$ \\
\hline$Z_{\text {hu }}{ }^{24}$ & 2016 & 0.521 & $396 / 378$ & $|3|$ & 194 & 71 & $456(57.6)$ & $336(42.4)$ & 154 & 179 & 45 & $487(64.4)$ & $269(35.6)$ \\
\hline Luo et $\mathrm{a}^{25}$ & 2017 & 0.672 & $298 / 303$ & 129 & 130 & 39 & $388(65.1)$ & 208 (34.9) & 119 & 139 & 45 & $377(62.2)$ & $229(37.8)$ \\
\hline Zhu et $\mathrm{al}^{26}$ & 2017 & 0.085 & $523 / 510$ & 170 & 267 & 86 & $607(58.0)$ & $439(42.0)$ & 204 & 251 & 55 & $659(64.6)$ & $361(35.4)$ \\
\hline \multicolumn{4}{|c|}{ miR-| $96 \mathrm{a} 2$ (rs | $|6| 49 \mid 3)$} & TT & TC & CC & $\mathbf{T}$ & C & TT & TC & CC & $\mathbf{T}$ & C \\
\hline Jeon et $\mathrm{al}^{16}$ & 2013 & 0.126 & $678 / 553$ & 139 & 352 & 187 & $630(46.5)$ & $726(53.5)$ & 105 & 292 & 156 & $502(45.4)$ & $604(54.6)$ \\
\hline Liu et $\mathrm{a}^{18}$ & 2014 & 0.060 & $296 / 391$ & 51 & $|8|$ & 64 & $283(47.8)$ & $309(52.2)$ & 84 & 214 & 93 & $382(48.8)$ & $400(51.2)$ \\
\hline Zhu et $\mathrm{al}^{19}$ & 2014 & 0.384 & $368 / 381$ & 71 & 189 & 108 & $331(45.0)$ & $405(55.0)$ & 78 & 198 & 105 & $354(46.5)$ & $408(53.5)$ \\
\hline Huang et $\mathrm{a}^{20}$ & 2015 & 0.856 & $53|/ 53|$ & 100 & 265 & 166 & $465(43.8)$ & $597(56.2)$ & 112 & 266 & 153 & $490(46.1)$ & $572(53.9)$ \\
\hline Zhu $^{24}$ & 2016 & 0.354 & $396 / 378$ & 112 & 205 & 79 & $429(54.2)$ & $363(45.8)$ & 110 & 196 & 72 & $416(55.0)$ & $340(45.0)$ \\
\hline Luo et $\mathrm{a}^{25}$ & 2017 & 0.385 & $298 / 303$ & 73 & 138 & 87 & $284(47.7)$ & $312(52.3)$ & 75 & 159 & 69 & $309(51.0)$ & $297(49.0)$ \\
\hline Zhu et $\mathrm{al}^{26}$ & 2017 & 0.548 & $523 / 510$ & 150 & 273 & 100 & $573(54.8)$ & $473(45.2)$ & 146 & 260 & 104 & $552(54.1)$ & $468(45.9)$ \\
\hline \multicolumn{4}{|c|}{ miR-I 49 (rs2292832) } & TT & TC & CC & $\mathbf{T}$ & C & TT & TC & CC & $\mathbf{T}$ & C \\
\hline $\mathrm{He}$ and $\mathrm{Han}^{15}$ & 2013 & 0.303 & $357 / 373$ & 138 & 162 & 57 & $438(66.6)$ & $276(4 I .4)$ & 160 & 175 & 38 & $495(66.4)$ & $251(33.6)$ \\
\hline Jeon et $\mathrm{al}^{16}$ & 2013 & 0.921 & $678 / 553$ & 299 & 303 & 76 & $901(66.4)$ & $455(33.6)$ & 262 & 238 & 53 & $762(68.9)$ & $344(31.1)$ \\
\hline Hu et a $\left.\right|^{17}$ & 2014 & 0.199 & $196 / 205$ & 79 & 76 & 41 & $234(59.7)$ & $158(40.3)$ & 80 & 89 & 36 & $249(60.7)$ & $161(39.3)$ \\
\hline Zhu $^{24}$ & 2016 & 0.720 & $396 / 378$ & 165 & 179 & 52 & $509(64.3)$ & $283(35.7)$ & 190 & 158 & 30 & $538(7 I .2)$ & $218(28.8)$ \\
\hline Luo et $\mathrm{a}^{25}$ & 2017 & 0.447 & $298 / 303$ & $|3|$ & 127 & 40 & $389(65.3)$ & $207(34.7)$ & 121 & 136 & 46 & $378(62.4)$ & $228(37.6)$ \\
\hline Zhu et $\mathrm{al}^{26}$ & 2017 & 0.351 & $523 / 510$ & 232 & 221 & 70 & $685(65.5)$ & $361(34.5)$ & 240 & 213 & 57 & $693(67.9)$ & $327(32.1)$ \\
\hline \multicolumn{4}{|c|}{ miR-499 (rs3746444) } & AA & AG & GG & $\mathbf{A}$ & G & AA & AG & GG & A & G \\
\hline Jeon et $\mathrm{al}^{16}$ & 2013 & 0.740 & $678 / 553$ & 460 & 195 & 23 & $\mathrm{I}, \mathrm{II} 5(82.2)$ & $24 I(17.8)$ & 365 & 170 & 18 & $900(81.4)$ & $206(18.6)$ \\
\hline Liu et al ${ }^{18}$ & 2014 & 0.170 & $296 / 391$ & $|8|$ & 96 & 19 & $458(77.4)$ & $134(22.6)$ & 278 & 99 & 14 & $655(83.8)$ & $127(16.2)$ \\
\hline Huang et $\mathrm{a}^{20}$ & 2015 & 0.002 & $53|/ 53|$ & 398 & 133 & 0 & $929(87.5)$ & $133(12.5)$ & 403 & 128 & 0 & $934(87.9)$ & $128(12.1)$ \\
\hline Lyu et $\mathrm{a}^{23}$ & 2016 & 0.621 & $378 / 378$ & 257 & 110 & II & $624(82.5)$ & I 32 (I7.5) & 250 & 113 & 15 & $613(81.1)$ & $143(18.9)$ \\
\hline$Z_{\text {hu }}{ }^{24}$ & 2016 & 0.910 & $396 / 378$ & 255 & 123 & 18 & $633(79.9)$ & $159(20.1)$ & 249 & 116 & 13 & $614(81.2)$ & $142(18.8)$ \\
\hline Luo et $\mathrm{a}^{25}$ & 2017 & 0.131 & $298 / 303$ & 215 & 78 & 5 & $508(85.2)$ & $88(14.8)$ & 244 & 53 & 6 & $54 \mid(89.3)$ & $65(10.7)$ \\
\hline Zhu et $\mathrm{al}^{26}$ & 2017 & 0.380 & $505 / 510$ & 349 & 124 & 32 & $840(80.3)$ & $206(19.7)$ & 328 & 158 & 24 & $814(79.8)$ & $206(20.2)$ \\
\hline
\end{tabular}

Abbreviation: HWE, Hardy-Weinberg equilibrium.

Table 4 Overall meta-analysis of the association between ischemic stroke and polymorphisms in miR-I46a (rs29I0I64), miR-I96a2 (rsl I6I49I3), miR-I49 (rs2292832), and miR-499 (rs3746444)

\begin{tabular}{|c|c|c|c|c|c|c|}
\hline \multirow[t]{2}{*}{ Genetic model } & \multirow[t]{2}{*}{ OR $[95 \% \mathrm{Cl}]$} & \multirow[t]{2}{*}{ Z (P-value) } & \multicolumn{3}{|c|}{ Heterogeneity of study design } & \multirow{2}{*}{$\begin{array}{l}\text { Analysis } \\
\text { model }\end{array}$} \\
\hline & & & $\chi^{2}$ & df (P-value) & $I^{2}(\%)$ & \\
\hline \multicolumn{7}{|c|}{ miR-146a (rs2910164) from I3 case-control studies (5,726 cases and 7, I75 controls) } \\
\hline Allelic model (G-allele vs C-allele) & $1.10[0.99-1.22]$ & $1.74(0.08)$ & 47.91 & $12(<0.00 \mathrm{I})$ & 75 & Random \\
\hline Recessive model (GG vs GC+CC) & $1.20[1.02-1.42]$ & $2.16(0.03)$ & 31.55 & $12(0.002)$ & 62 & Random \\
\hline Dominant model (CC vs GC+GG) & $0.91[0.80-1.04]$ & $1.41(0.16)$ & 34.76 & $12(<0.00 \mathrm{I})$ & 65 & Random \\
\hline Homozygous model (GG vs CC) & $1.24[1.00-1.53]$ & $1.95(0.05)$ & 43.43 & $12(<0.00 \mathrm{I})$ & 72 & Random \\
\hline Heterozygous model (GC vs CC) & $1.06[0.95-1.17]$ & $1.00(0.32)$ & 20.79 & $12(0.05)$ & 42 & Random \\
\hline
\end{tabular}


Table 4 (Continued)

\begin{tabular}{|c|c|c|c|c|c|c|}
\hline \multirow[t]{2}{*}{ Genetic model } & \multirow[t]{2}{*}{ OR $[95 \% \mathrm{Cl}]$} & \multirow[t]{2}{*}{$Z$ (P-value) } & \multicolumn{3}{|c|}{ Heterogeneity of study design } & \multirow{2}{*}{$\begin{array}{l}\text { Analysis } \\
\text { model }\end{array}$} \\
\hline & & & $? ? 2$ & $d f(P$-value $)$ & $I^{2}(\%)$ & \\
\hline \multicolumn{7}{|c|}{ miR-I96a2 (rs I I6I49|3) from 7 case-control studies (3,090 cases and 3,047 controls) } \\
\hline Allelic model (C-allele vs T-allele) & $1.04[0.97-1.12]$ & $1.10(0.27)$ & 3.20 & $6(0.78)$ & 0 & Fixed \\
\hline Recessive model (CC vs TC+TT) & $1.04[0.93-1.17]$ & $0.73(0.46)$ & 4.60 & $6(0.60)$ & 0 & Fixed \\
\hline Dominant model (TT vs TC+CC) & $0.95[0.85-1.08]$ & $0.77(0.44)$ & 2.86 & $6(0.83)$ & 0 & Fixed \\
\hline Homozygous model (CC vs TT) & $1.07[0.92-1.24]$ & $0.91(0.36)$ & 2.85 & $6(0.83)$ & 0 & Fixed \\
\hline Heterozygous model (TC vs TT) & $1.07[0.93-1.23]$ & $0.90(0.37)$ & 2.72 & $5(0.74)$ & 0 & Fixed \\
\hline \multicolumn{7}{|c|}{ miR-I 49 ( $r$ 22292832) from 6 case-control studies (2,448 cases and 2,322 controls) } \\
\hline Allelic model (C-allele vs T-allele) & $1.09[1.00-1.18]$ & $1.91(0.06)$ & 4.84 & $5(0.44)$ & 0 & Fixed \\
\hline Recessive model (CC vs TC+TT) & $1.28[1.08-1.52]$ & $2.80(0.005)$ & 6.14 & $5(0.29)$ & 19 & Fixed \\
\hline Dominant model (TT vs TC+CC) & $0.89[0.79-1.00]$ & $1.99(0.05)$ & 6.31 & $5(0.28)$ & 21 & Fixed \\
\hline Homozygous model (CC vs TT) & $1,31[1.09-1.58]$ & $2.92(0.004)$ & 8.27 & $5(0.14)$ & 40 & Fixed \\
\hline Heterozygous model (TC vs TT) & $1.07[0.95-1.21]$ & $1.12(0.26)$ & 4.22 & $5(0.52)$ & 0 & Fixed \\
\hline \multicolumn{7}{|c|}{ miR-499 (rs3746444) from 7 case-control studies (3,082 cases and 3,044 controls) } \\
\hline Allelic model (G-allele vs A-allele) & $1.09[0.95-1.25]$ & $1.28(0.20)$ & 12.36 & $6(0.05)$ & 51 & Random \\
\hline Recessive model (GG vs $A G+A A)$ & $1.21[0.91-1.61]$ & $1.31(0.19)$ & 3.81 & $5(0.58)$ & 0 & Fixed \\
\hline Dominant model (AA vs $A G+G G)$ & $0.93[0.78-1.12]$ & $0.77(0.44)$ & 16.43 & $6(0.01)$ & 63 & Random \\
\hline Homozygous model (GG vs AA) & $1.20[0.90-1.60]$ & $\mathrm{I} .25(0.2 \mathrm{I})$ & 4.47 & $5(0.48)$ & 0 & Fixed \\
\hline Heterozygous model (AG vs AA) & $1.06[0.87-1.28]$ & $0.56(0.57)$ & 17.10 & $6(0.009)$ & 65 & Random \\
\hline
\end{tabular}

\section{IS risk and miR-196a2 (rs I 6 |49|3) polymorphism}

The overall results are summarized in Table 4 and Figure 3. On the basis of 3,090 cases and 3,047 controls from seven studies, ${ }^{16,18-20,24-26}$ miR-196a2 (rs11614913) polymorphism did not show significant association with IS risk in any of the following five genetic models: allelic model, $\mathrm{OR}=1.04$, 95\% CI=0.97-1.12, $P=0.27$ (Figure 3A); recessive model, $\mathrm{OR}=1.04,95 \% \mathrm{CI}=0.93-1.17, P=0.46$ (Figure 3B); dominant model, OR=0.95, 95\% CI=0.85-1.08, $P=0.44$ (Figure 3C); homozygous model, $\mathrm{OR}=0.95,95 \% \mathrm{CI}=0.85-1.08, P=0.44$ (Figure 3D); and heterozygous model, OR=1.07, 95\% $\mathrm{CI}=0.93-1.23, P=0.37$ (Figure 3E).

\section{IS risk and miR-I49 (rs2292832) polymorphism}

The overall results for miR-149 (rs2292832) are summarized in Table 4 and Figure 4. On the basis of 2,448 cases and
A

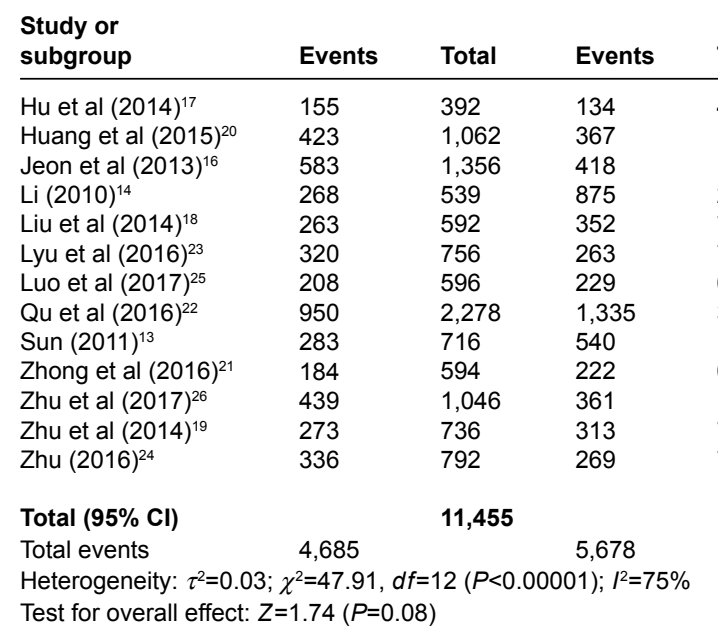

Figure 2 (Continued)

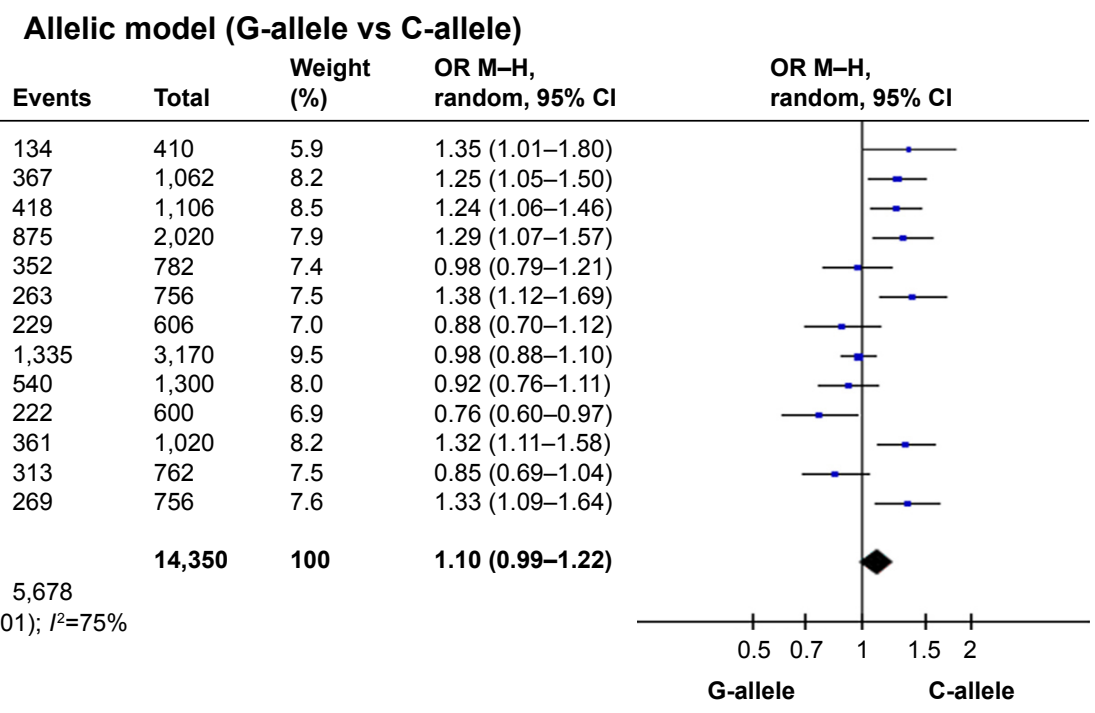

OR M-H

random, $95 \% \mathrm{Cl}$

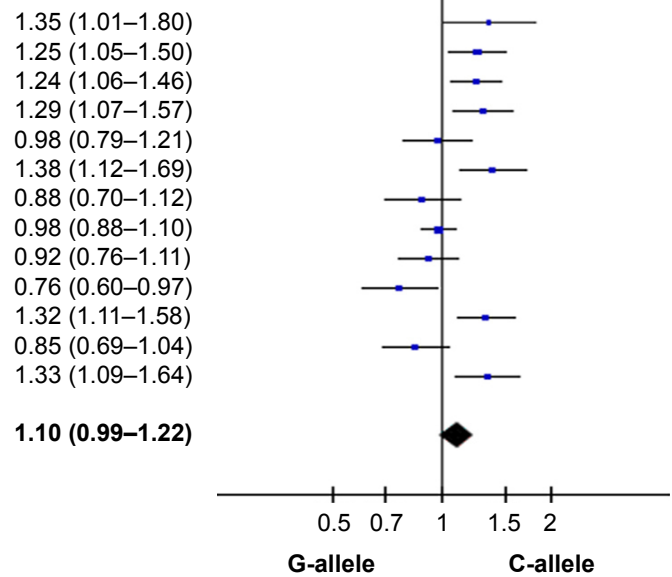


B

\begin{tabular}{|c|c|c|c|c|c|c|c|c|c|c|}
\hline \multirow{2}{*}{$\begin{array}{l}\text { Study or } \\
\text { subgroup }\end{array}$} & \multicolumn{9}{|c|}{ Recessive model (GG vs GC+CC) } & \\
\hline & \multirow{2}{*}{$\begin{array}{l}\text { Events } \\
34\end{array}$} & \multirow{2}{*}{$\begin{array}{l}\text { Total } \\
196\end{array}$} & \multirow{2}{*}{$\begin{array}{l}\text { Events } \\
26\end{array}$} & \multirow{2}{*}{$\begin{array}{l}\text { Total } \\
205\end{array}$} & \multirow{2}{*}{$\begin{array}{l}\text { Weight } \\
\text { (\%) }\end{array}$} & \multirow{2}{*}{$\begin{array}{l}\text { OR } \begin{array}{l}\mathrm{M}-\mathrm{H}, \\
\text { random, } 95 \% \mathrm{CI}\end{array} \\
1.44(0.83-2.51)\end{array}$} & \multicolumn{4}{|c|}{$\begin{array}{l}\text { OR } \mathrm{M}-\mathrm{H} \text {, } \\
\text { random, } 95 \% \mathrm{Cl}\end{array}$} \\
\hline Hu et al $(2014)^{17}$ & & & & & & & & & & \\
\hline Huang et al $(2015)^{20}$ & 81 & 531 & 55 & 531 & 8.0 & $1.56(1.08-2.25)$ & & & & \\
\hline Jeon et al $(2013)^{16}$ & 128 & 678 & 76 & 553 & 9.0 & $1.46(1.07-1.99)$ & & & $=$ & \\
\hline $\mathrm{Li}(2010)^{14}$ & 79 & 268 & 210 & 1,010 & 9.1 & $1.59(1.18-2.16)$ & & & $\longrightarrow$ & \\
\hline Liu et al $(2014)^{18}$ & 52 & 296 & 77 & 391 & 7.6 & $0.87(0.59-1.28)$ & & & - & \\
\hline Lyu et al $(2016)^{23}$ & 61 & 378 & 38 & 378 & 6.9 & $1.72(1.12-2.85)$ & & & - & \\
\hline Luo et al $(2017)^{25}$ & 39 & 298 & 45 & 303 & 6.5 & $0.86(0.54-1.37)$ & & & - & \\
\hline Qu et al $(2016)^{22}$ & 166 & 1,139 & 233 & 1,585 & 10.7 & $0.99(0.80-1.23)$ & & & . & \\
\hline Sun $(2011)^{13}$ & 61 & 358 & 118 & 650 & 8.4 & $0.93(0.66-1.30)$ & & & & \\
\hline Zhong et al (2016) & 28 & 297 & 35 & 300 & 5.7 & $0.79(0.47-1.33)$ & & & - & \\
\hline Zhu et al $(2017)^{26}$ & 86 & 523 & 55 & 510 & 8.0 & $1.63(1.13-2.34)$ & & & $\longrightarrow$ & \\
\hline Zhu et al $(2014)^{19}$ & 50 & 368 & 64 & 381 & 7.4 & $0.78(0.52-1.16)$ & & & - & \\
\hline Zhu $(2016)^{24}$ & 71 & 396 & 45 & 378 & 7.4 & $1.62(1.08-2.42)$ & & & & \\
\hline Total $(95 \% \mathrm{Cl})$ & & 5,726 & & 7,175 & 100 & $1.20(1.02-1.42)$ & & & & \\
\hline Total events & 936 & & 1,077 & & & & & & & \\
\hline \multicolumn{7}{|c|}{ Heterogeneity: $\tau^{2}=0.06 ; \chi^{2}=31.55, d f=12(P=0.002) ; l^{2}=62 \%$} & +1 & 1 & & + \\
\hline \multirow{2}{*}{\multicolumn{7}{|c|}{ Test for overall effect: $Z=2.16(P=0.03)$}} & $0.1 \quad 0.2$ & 0.5 & 2 & 10 \\
\hline & & & & & & & \multicolumn{4}{|c|}{ GG } \\
\hline
\end{tabular}

C

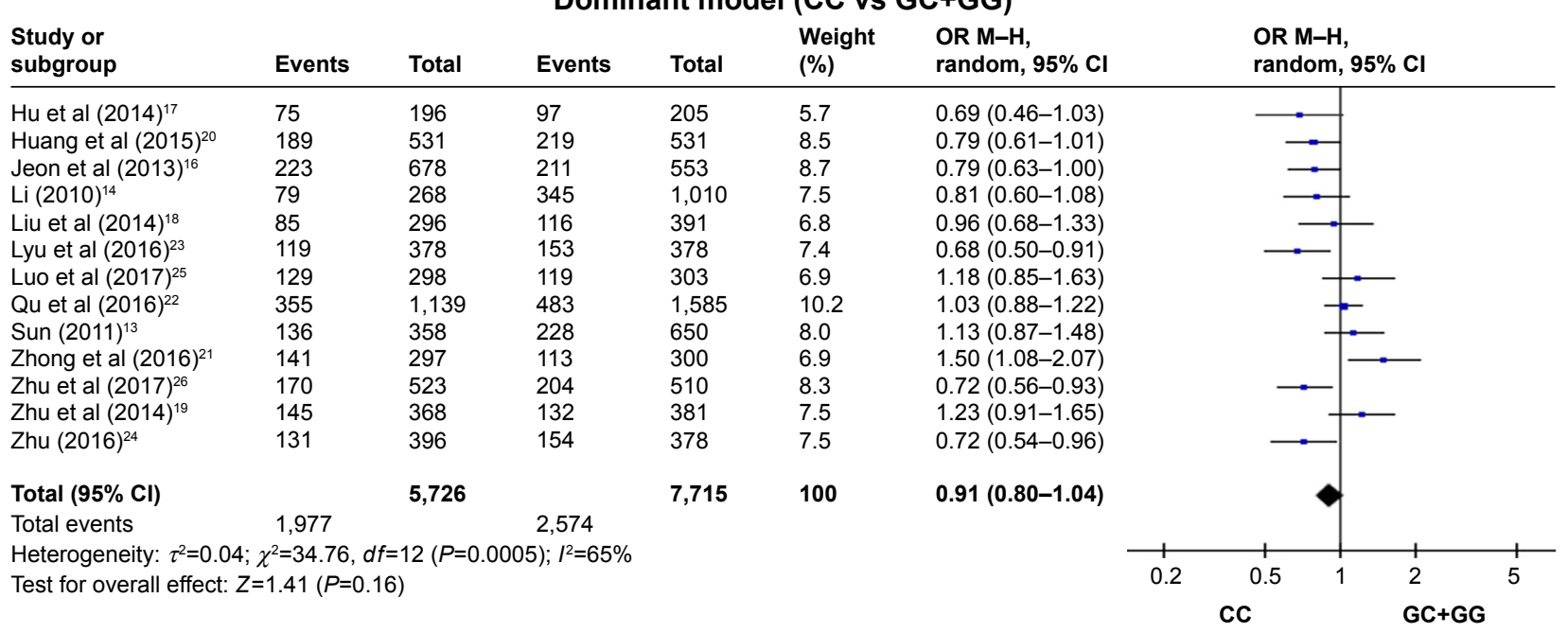

D

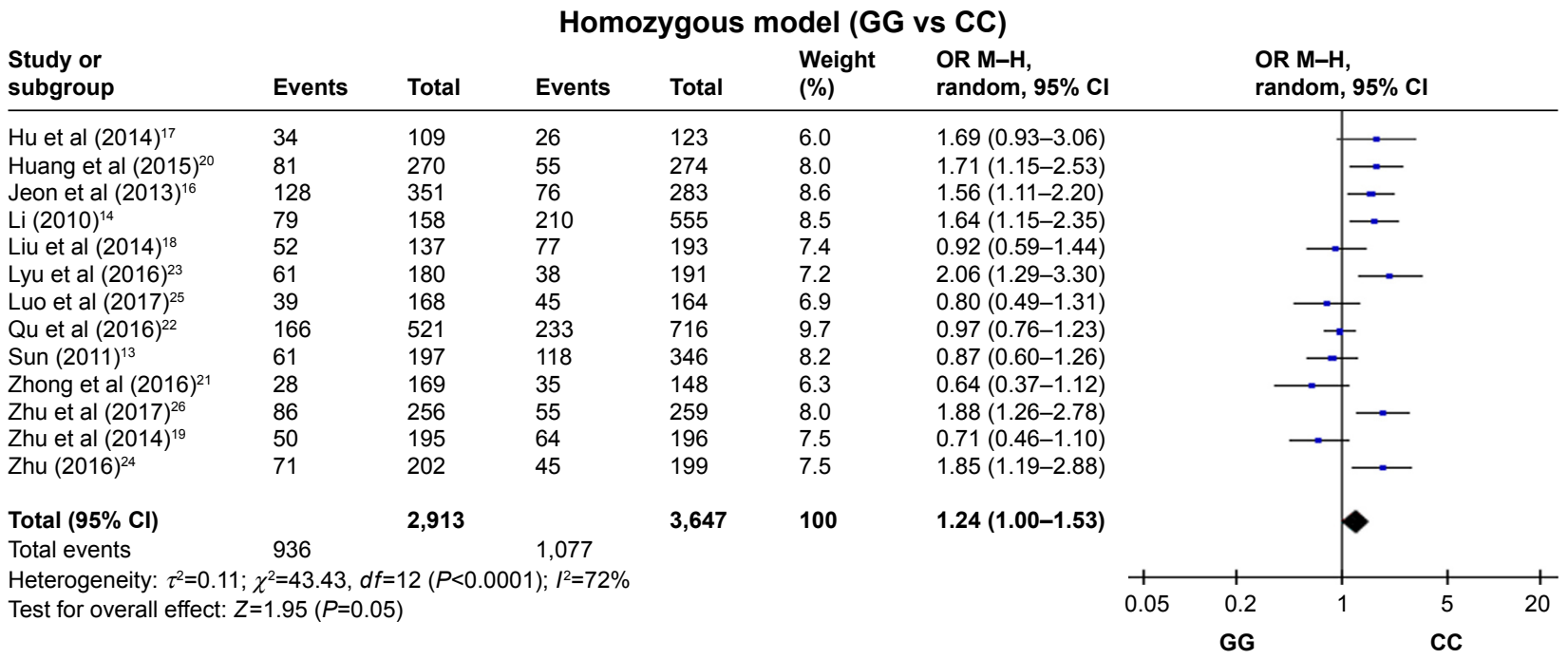

Figure 2 (Continued) 
E

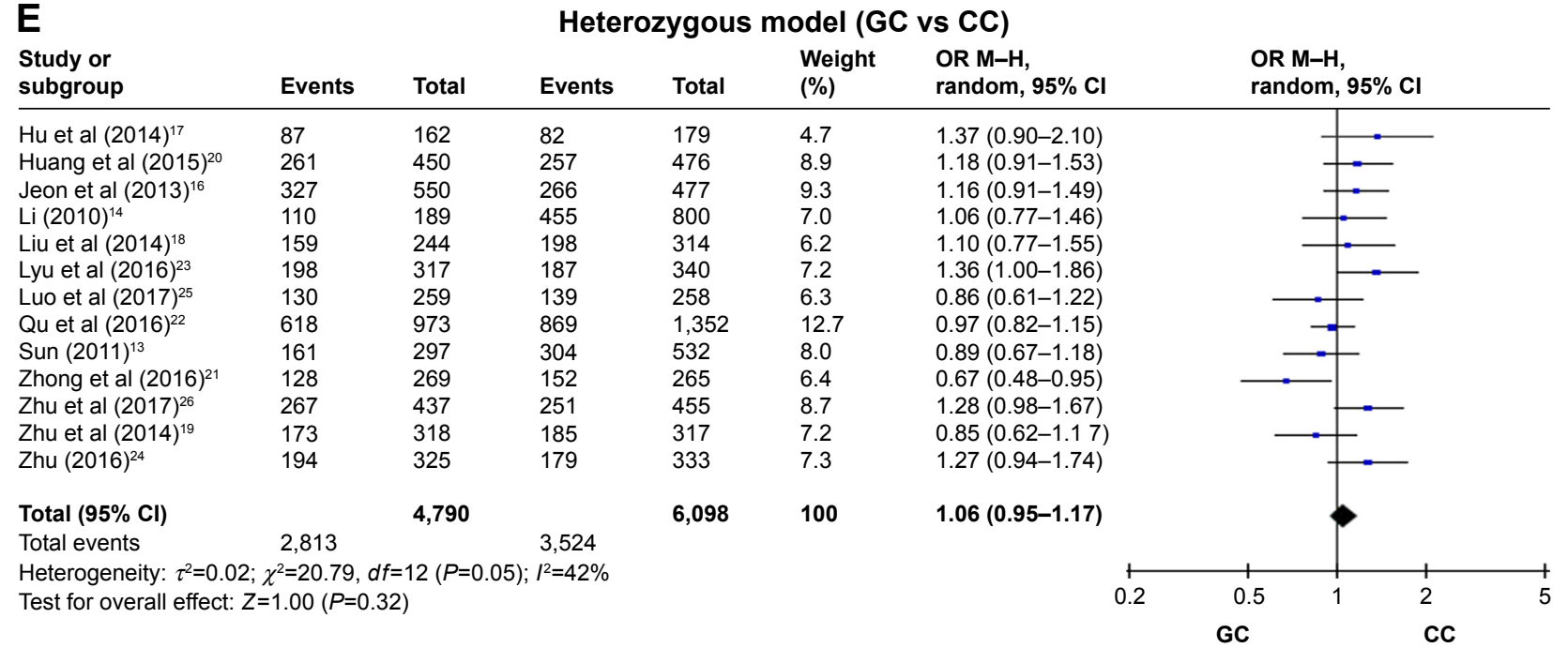

Figure 2 Forest plot describing the association between the miR-146a (rs2910164) polymorphism and ischemic stroke risk according to different genetic models: (A) allelic (G-allele vs C-allele), (B) recessive (GG vs GC+CC), (C) dominant (CC vs GC+GG), (D) homozygous (GG vs CC), and (E) heterozygous (GC vs CC).

A

\begin{tabular}{llll}
$\begin{array}{l}\text { Study or } \\
\text { subgroup }\end{array}$ & Events & Total & Events \\
\hline $\begin{array}{l}\text { Huang et al }(2015)^{20} \\
\text { Jeon et al }(2013)^{16}\end{array}$ & 726 & 1,062 & 572 \\
Liu et al $(2014)^{18}$ & 309 & 592 & 400 \\
Luo et al $(2017)^{25}$ & 312 & 596 & 297 \\
Zhu et al $(2017)^{26}$ & 413 & 1,046 & 468 \\
Zhu et al $(2014)^{19}$ & 405 & 736 & 408 \\
Zhu $(2016)^{24}$ & 363 & 792 & 340 \\
& & & \\
Total (95\% Cl) & & $\mathbf{6 , 1 8 0}$ & \\
Total events & 3,185 & & 3,069 \\
Heterogeneity: $\chi^{2}=3.20, d f=6(P=0.78) ; I^{2}=0 \%$
\end{tabular}

Allelic model (C-allele vs T-allele)

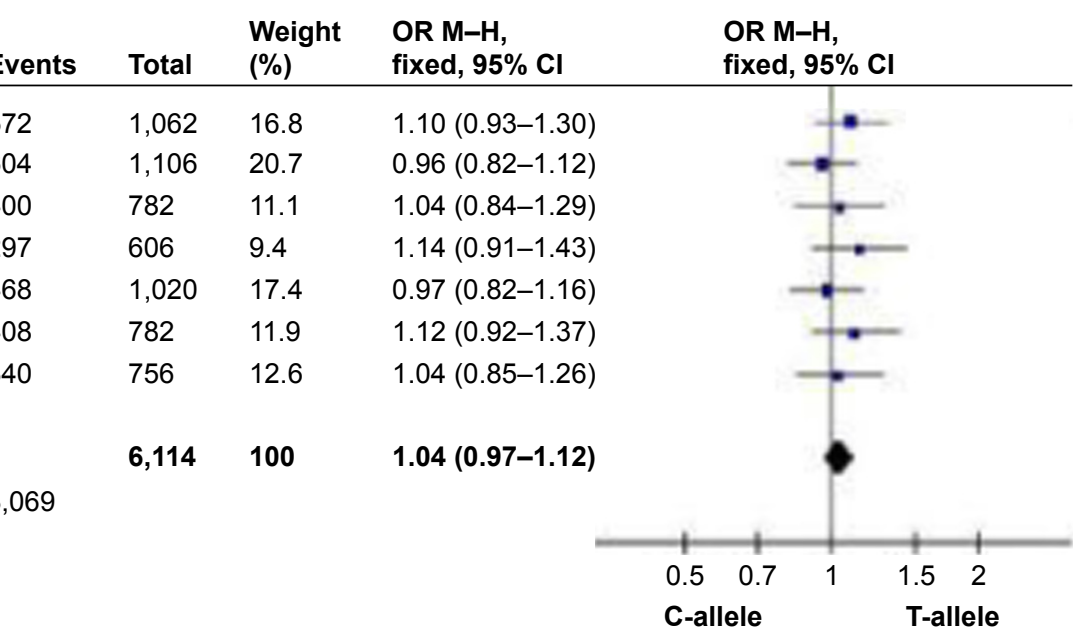

B

\begin{tabular}{llll}
$\begin{array}{l}\text { Study or } \\
\text { subgroup }\end{array}$ & Events & Total & Event \\
\hline Huang et al $(2015)^{20}$ & 166 & 531 & 153 \\
Jeon et al $(2013)^{16}$ & 187 & 678 & 156 \\
Liu et al $(2014)^{18}$ & 64 & 296 & 93 \\
Luo et al $(2017)^{25}$ & 87 & 298 & 69 \\
Zhu et al $(2017)^{26}$ & 100 & 523 & 104 \\
Zhu et al $(2014)^{19}$ & 108 & 368 & 105 \\
Zhu $(2016)^{24}$ & 79 & 396 & 72 \\
& & & \\
Total (95\% Cl) & & 3,090 & \\
Total events & 791 & & 752 \\
Heterogeneity: $\chi^{2}=4.60, d f=6(P=0.60) ; l^{2}=0 \%$ \\
Test for overall effect: $Z=0.73(P=0.46)$
\end{tabular}
752
Recessive model (CC vs TC+TT)

$\begin{array}{llll} & \text { Weight } & \mathrm{OR} \mathrm{M}-\mathrm{H}, & \mathrm{OR} \mathrm{M}-\mathrm{H} \text {, }\end{array}$
fixed, $95 \%$ C $1.12(0.86-1.46)$ $0.97(0.75-1.24)$ $0.88(0.62-1.27)$ $1.40(0.97-2.02)$ $0.92(0.68-1.25)$ $1.09(0.79-1.50)$ $1.06(0.74-1.51)$ $1.04(0.93-1.17)$

$3,047 \quad 100$

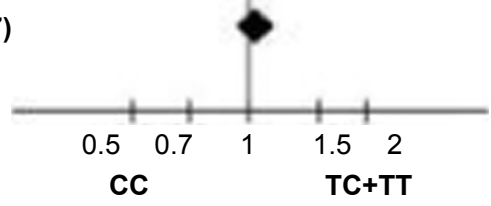

Figure 3 (Continued) 
C

Study or
subgroup

\begin{tabular}{lllll} 
& Events & Total & Events & Total \\
\hline Huang et al $(2015)^{20}$ & 100 & 531 & 112 & 531
\end{tabular}

Jeon et al $(2013)^{16} \quad 139 \quad 678$

Liu et al $(2014)^{18} \quad 51 \quad 296$

Luo et al $(2017)^{25} \quad 73 \quad 298$

Zhu et al $(2017)^{26} \quad 150 \quad 523$

Zhu et al (2014) $)^{19}$

Zhu $(2016)^{24}$

71

112

368

396

105

84

75

146

78

110

3,090

Total $(95 \% \mathrm{Cl})$

Total events

696

Heterogeneity: $\chi^{2}=2.86, d f=6(P=0.83) ; l^{2}=0 \%$

Test for overall effect: $Z=0.77(P=0.44)$

710
Dominant model (TT vs TC+CC)

Weight OR M-H,

(\%) fixed, $95 \% \mathrm{CI}$

$0.37(0.64-1.17)$

$1.10(0.83-1.46)$

$0.76(0.52-1.12)$

$0.99(0.68-1.43)$

$1.00(0.77-1.31)$

$0.93(0.65-1.33)$

$0.96(0.70-1.31)$

$0.96(0.85-1.08)$

$3,047 \quad 100$

100

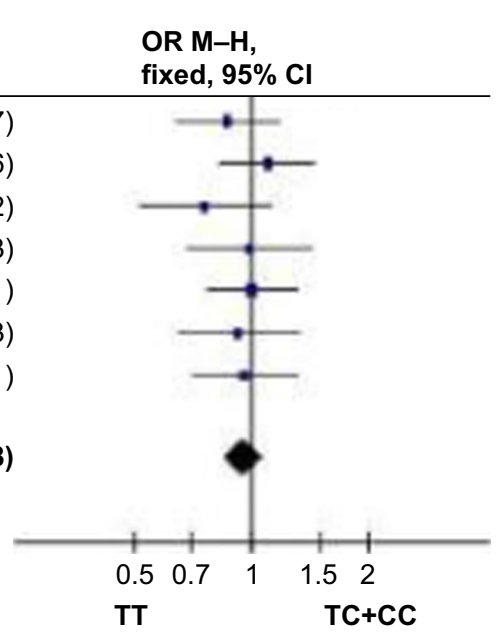

D Homozygous model (CC vs TT)

Study or subgroup Huang et al $(2015)^{20}$ Jeon et al $(2013)^{16}$ Liu et al (2014) ${ }^{18}$ Luo et al $(2017)^{25}$ Zhu et al (2017) $)^{26}$ Zhu et al (2014) ${ }^{19}$ Zhu (2016) $)^{24}$

Total $(95 \% \mathrm{Cl})$

Total events

791

1,487

Events Total

Weight OR M-H,

(\%) fixed, $95 \% \mathrm{Cl}$

$1.22(0.86-1.72)$

$156 \quad 261 \quad 21.5$

$0.91(0.65-1.26)$

$187 \quad 326$

261
177

21.5

$1.13(0.71-1.82)$

$\begin{array}{ll}64 & 115 \\ 87 & 160\end{array}$

93

144

9.6

$1.30(0.82-2.03)$

$100 \quad 250$

104

250

18.1

$0.94(0.66-1.34)$

$108 \quad 179$

105

183

12.0

$1.13(0.74-1.72)$

$1.08(0.71-1.63)$

Heterogeneity: $\chi^{2}=2.85, d f=6(P=0.83) ; I^{2}=0 \%$

Test for overall effect: $Z=0.91(P=0.36)$

$1,462 \quad 100$

$1.07(0.92-1.24)$

752

1,462

100

4)

OR $\mathrm{M}-\mathrm{H}$, fixed, $95 \% \mathrm{CI}$

Heterozygous model (TC vs TT)

\begin{tabular}{|c|c|c|c|c|c|c|c|}
\hline $\begin{array}{l}\text { Study or } \\
\text { subgroup }\end{array}$ & Events & Total & Events & Total & $\begin{array}{l}\text { Weight } \\
(\%)\end{array}$ & $\begin{array}{l}\text { OR } \mathrm{M}-\mathrm{H} \text {, } \\
\text { fixed, } 95 \% \mathrm{Cl}\end{array}$ & $\begin{array}{l}\text { OR M-H, } \\
\text { fixed, } 95 \% \text { CI }\end{array}$ \\
\hline Huang et al $(2015)^{20}$ & 265 & 365 & 266 & 378 & 18.7 & $1.12(0.81-1.53)$ & - \\
\hline Jeon et al $(2013)^{16}$ & 352 & 291 & 292 & 397 & & Not estimable & \\
\hline Liu et al $(2014)^{18}$ & 181 & 232 & 214 & 298 & 10.8 & $1.39(0.93-2.08)$ & \\
\hline Luo et al $(2017)^{25}$ & 138 & 211 & 159 & 234 & 13.7 & $0.89(0.60-1.32)$ & \\
\hline Zhu et al $(2017)^{26}$ & 273 & 423 & 260 & 406 & 24.6 & $1.02(0.77-1.36)$ & \\
\hline Zhu et al $(2014)^{19}$ & 189 & 260 & 198 & 275 & 13.7 & $1.05(0.72-1.53)$ & \\
\hline Zhu $(2016)^{24}$ & 205 & 317 & 196 & 306 & 18.4 & $1.03(0.74-1.43)$ & \\
\hline Total $(95 \% \mathrm{Cl})$ & & 2,099 & & 2,295 & 100 & $1.07(0.93-1.23)$ & \\
\hline Total events & 1,603 & & 1,585 & & & & \\
\hline \multicolumn{7}{|c|}{$\begin{array}{l}\text { Heterogeneity: } \chi^{2}=2.72, d f=6(P=0.74) ; l^{2}=0 \% \\
\text { Test for overall effect: } Z=0.90(P=0.37)\end{array}$} & $\begin{array}{lll}0.5 & 0.7 & 1 \\
\text { TC } & \end{array}$ \\
\hline
\end{tabular}

Figure 3 Forest plot describing the association between the miR-196a2 (rs/16/49/3) polymorphism and ischemic stroke risk according to different genetic models: (A) allelic, (B) recessive, (C) dominant, (D) homozygous, and (E) heterozygous. 
2,322 controls from six studies, ${ }^{16,18,20,23-26}$ the overall results indicated that the CC genotype of miR-149 (rs2292832) may be associated with increased IS risk according to the recessive model $(\mathrm{OR}=1.28,95 \% \mathrm{CI}=1.08-1.52, P=0.005$; Figure 4B) and homozygous model $(\mathrm{OR}=1.31,95 \% \mathrm{CI}=1.09-1.58$, $P=0.004$; Figure 4D).

\section{IS risk and miR-499 (rs3746444) polymorphism}

The overall results are summarized in Table 4 and Figure 5. On the basis of 3,082 cases and 3,044 controls from seven studies, ${ }^{16,18,20,23-26}$ miR-499 (rs3746444) polymorphism did not show significant association with IS risk in any of the following five genetic models: allelic model, $\mathrm{OR}=1.09$, 95\% CI=0.95-1.25, $P=0.20$ (Figure 5A); recessive model, $\mathrm{OR}=1.21,95 \% \mathrm{CI}=0.91-1.61, P=0.19$ (Figure 5B); dominant model, $\mathrm{OR}=0.93,95 \% \mathrm{CI}=0.78-1.12, P=0.44$ (Figure 5C); homozygous model, $\mathrm{OR}=1.20,95 \% \mathrm{CI}=0.90-1.60, P=0.21$
(Figure 5D); or heterozygous model, OR=1.06, 95\% $\mathrm{CI}=0.87-1.28, P=0.57$ (Figure 5E).

\section{Sensitivity analysis}

Sensitivity analysis was conducted for miR-146a (rs2910164) by excluding the studies by $\mathrm{Li}$ et $\mathrm{al}^{14}$ and $\mathrm{Qu}$ et al; ${ }^{22}$ the $P$-value for HWE was less than 0.05 for these two studies. The recessive model gave different results $(\mathrm{OR}=1.19$, 95\% CI $=0.98-1.45, P=0.07$ ) than those obtained when all studies were meta-analyzed. Sensitivity analysis was conducted for miR-146a (rs2910164) by excluding one study by Jeon et al. ${ }^{16}$ Again, the recessive model gave different results $(\mathrm{OR}=1.18,95 \% \mathrm{CI}=0.99-1.41, P=0.07)$ than when all studies were included. Therefore, the results for miR-146a (rs2910164) should be interpreted with caution.

Sensitivity analysis was conducted for miR-196a2 (rs11614913) by excluding the study by Jeon et al. ${ }^{16}$ The results were similar to those obtained with all studies,

A

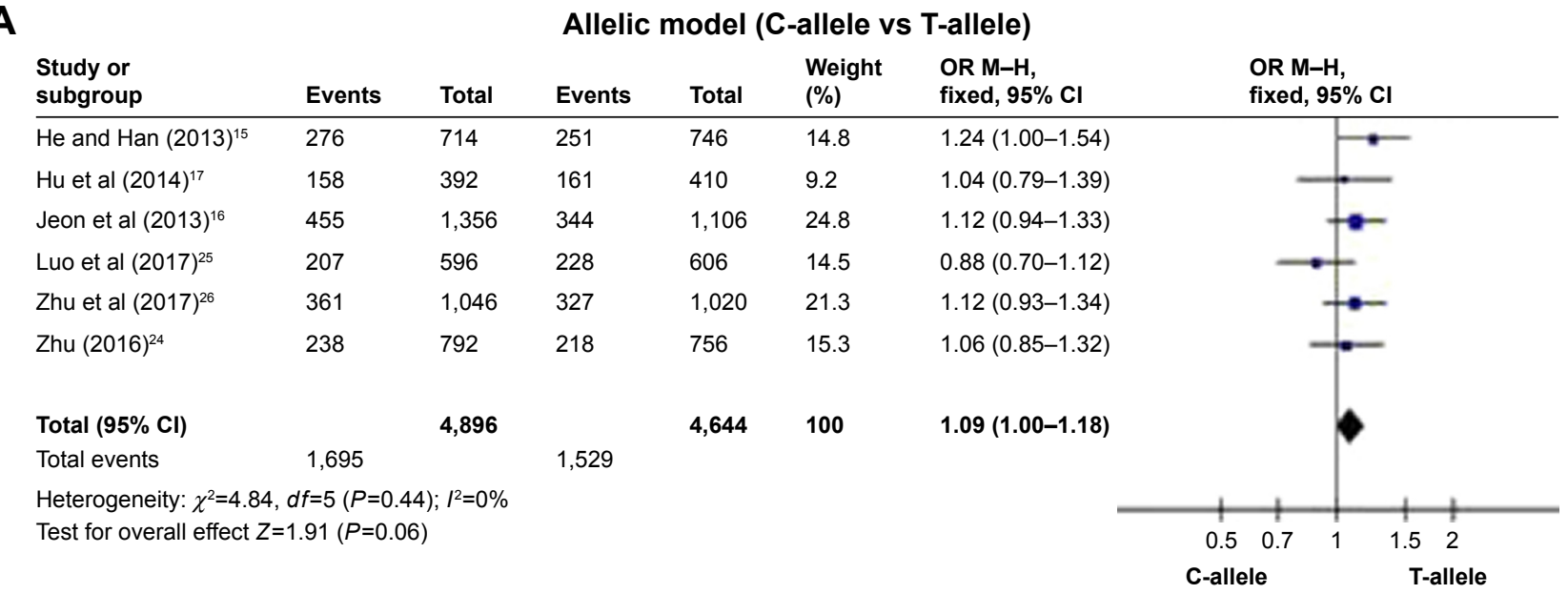

B

\begin{tabular}{llll}
$\begin{array}{l}\text { Study or } \\
\text { subgroup }\end{array}$ & Events & Total & Events \\
\hline He and Han $(2013)^{15}$ & 57 & 357 & 38 \\
Hu et al $(2014)^{17}$ & 41 & 196 & 36 \\
Jeon et al $(2013)^{16}$ & 76 & 678 & 53 \\
Luo et al $(2017)^{25}$ & 40 & 298 & 46 \\
Zhu et al $(2017)^{26}$ & 70 & 523 & 57 \\
Zhu (2016) ${ }^{24}$ & 52 & 396 & 30 \\
& & & \\
Total (95\% CI) & & 2,448 & \\
Total events & 336 & & \\
Heterogeneity: $\chi^{2}=6.14, d f=5(P=0.29) ; I^{2}=19 \%$ & \\
Test for overall effect $Z=2.80(P=0.005)$ &
\end{tabular}

\section{Recessive model (CC vs TC+TT)}

$\begin{array}{lll} & \text { Weight } \quad \text { OR M-H, OR M-H }\end{array}$

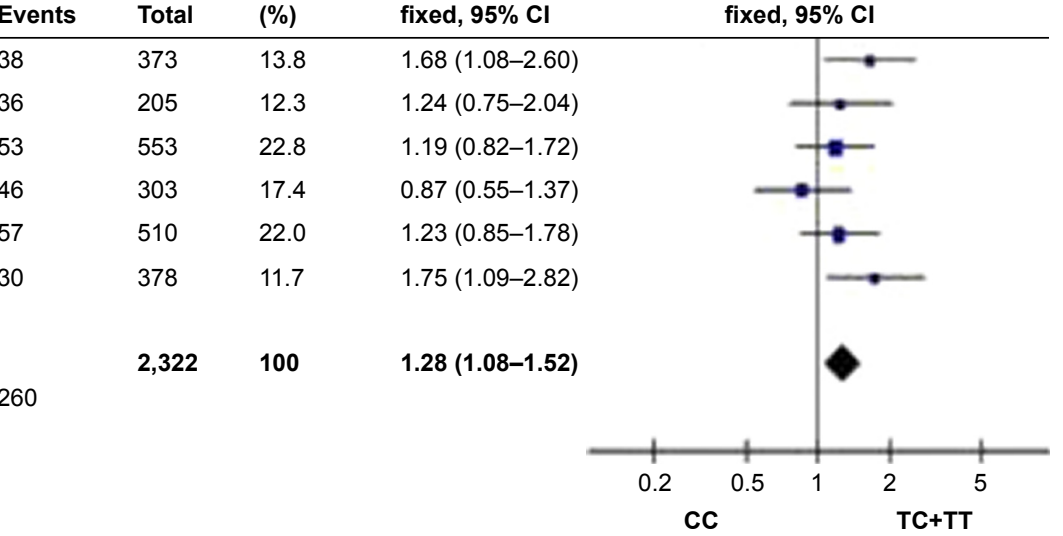

Figure 4 (Continued) 


\section{C}

Dominant model (TT vs TC+CC)

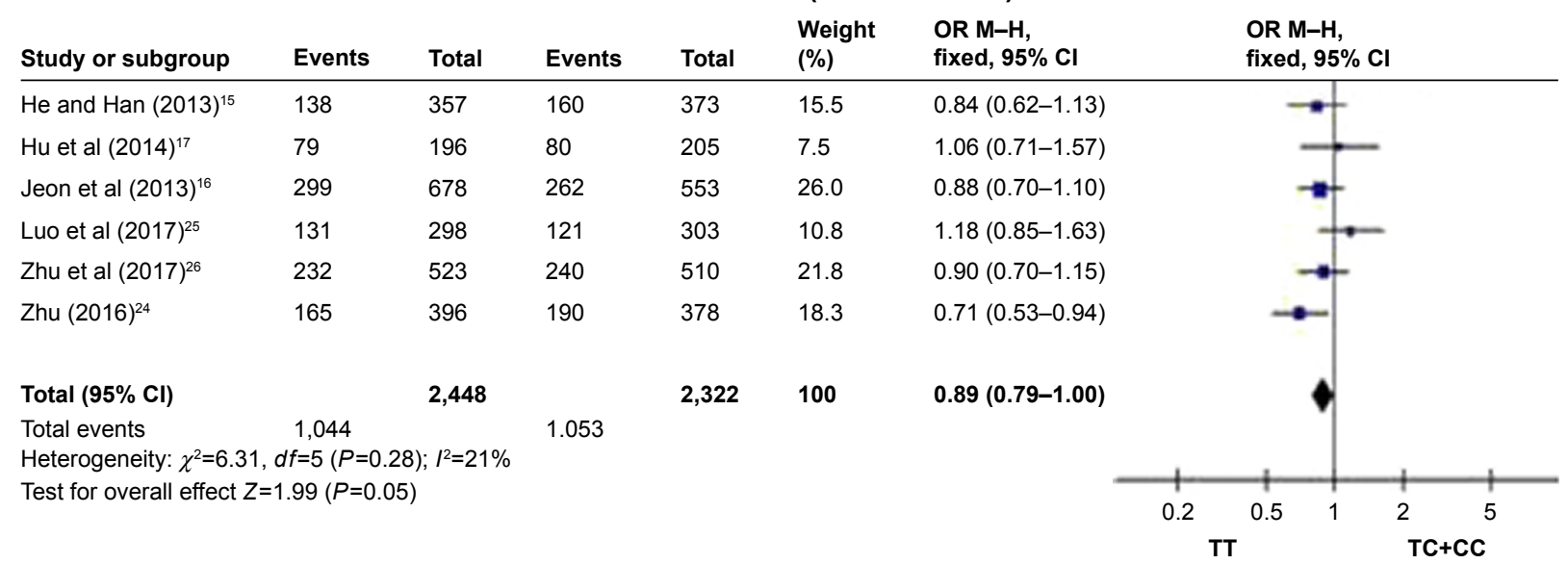

D

\section{Homozygous model (CC vs TT)}

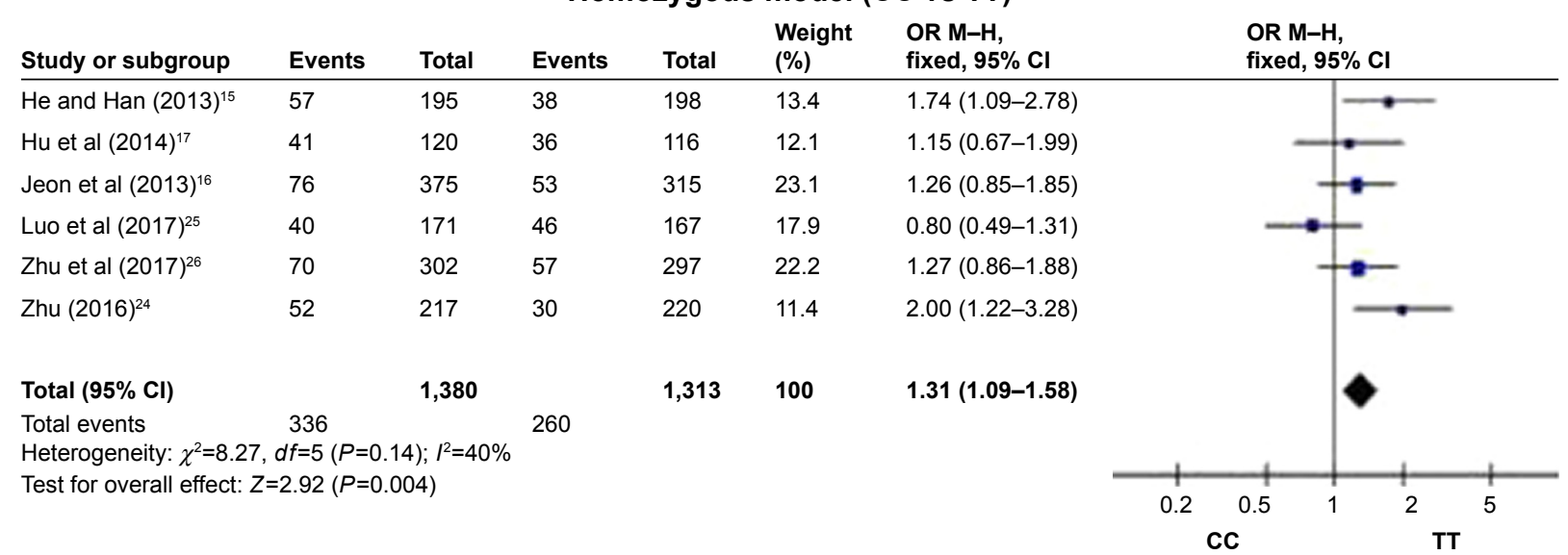

E

Heterozygous model (TC vs TT)

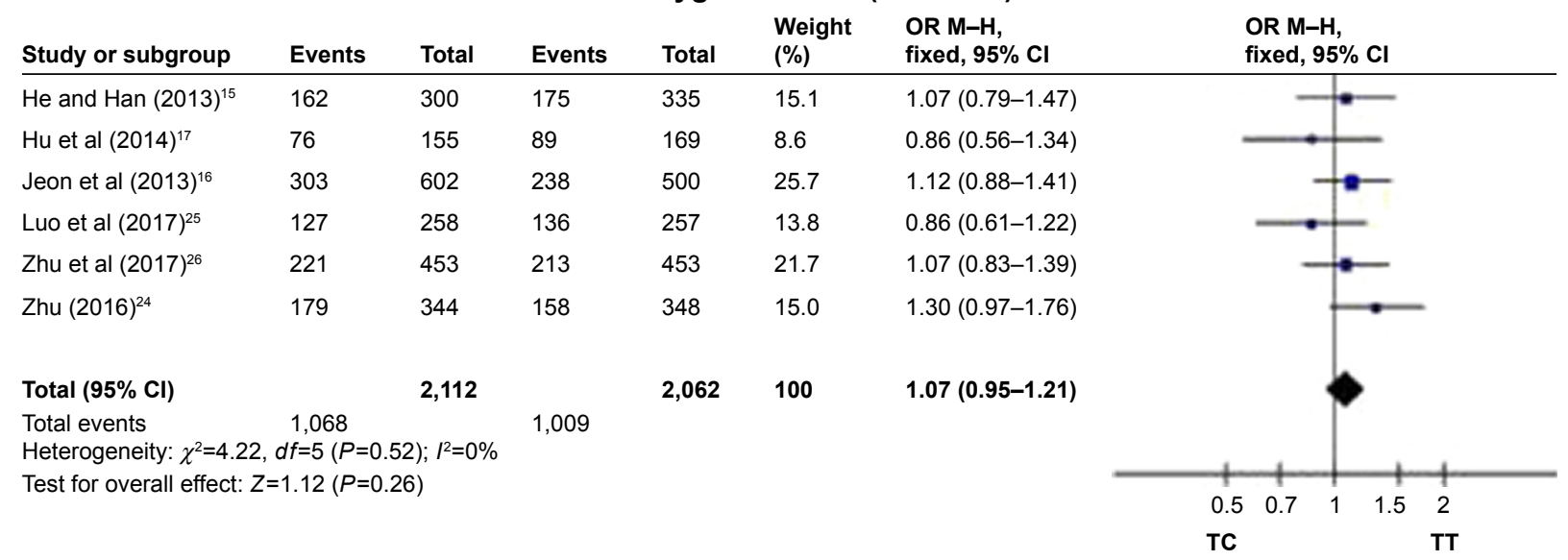

Figure 4 Forest plot describing the association between the miR-I49 (rs2292832) polymorphism and ischemic stroke risk according to different genetic models: (A) allelic, (B) recessive, (C) dominant, (D) homozygous, and (E) heterozygous.

regardless of the genetic model. This implies that our metaanalysis results for miR-196a2 (rs11614913) are robust. Similar robustness was observed when we performed sensitivity analysis for miR-149 (rs2292832) and for miR-499 (rs3746444) by excluding the study by Jeon et al. ${ }^{16}$

Sensitivity analysis was conducted for miR-499 (rs3746444) by excluding a study by Huang et al, ${ }^{20}$ in which the $P$-value of HWE was less than 0.05 . The results were not altered in any of the five genetic models.

\section{Publication bias}

Begg's funnel plot and Egger's test were performed to detect potential publication bias in this meta-analysis. No obvious asymmetry was observed in Begg's funnel plots in 
the recessive model, and Egger's tests (Figure 6) indicated no publication bias.

\section{Discussion}

Previous studies have demonstrated that mutations in the pre-miRNA of miR-146a, miR-499, miR-149, and miR$196 \mathrm{a} 2$ decrease the levels of the corresponding mature miRNAs. ${ }^{20,29,30}$ These four miRNAs affect thrombosis or inflammation pathways in the circulatory system by regulating tumor necrosis factor- $\alpha$ (TNF- $\alpha),{ }^{31}$ methylenetetrahydrofolate reductase, ${ }^{32}$ annexin A1, ${ }^{33} \mathrm{C}$-reactive protein, ${ }^{34}$ the NF- $\kappa$ B pathway, and the MAP kinase pathway. ${ }^{35}$ Many studies have been conducted to reveal the impact of SNPs on precursor and mature miRNAs and their associations with IS risk. ${ }^{13-26}$ In fact, several meta-analyses have been conducted to explore the association between miRNA polymorphisms and IS risk. The results have been inconsistent, largely due to limited sample size. ${ }^{36-39}$ Therefore, we conducted this metaanalysis on all eligible studies to provide a more precise estimate of the association of IS risk with miR-146a (rs2910164), miR-196a2 (rs11614913), miR-149 (rs2292832), and miR499 (rs3746444). Interestingly, all the case-control studies in our meta-analysis analyzed Asian populations.

A previous meta-analysis by Zhu et $\mathrm{al}^{39}$ found the $\mathrm{C}$ allele of miR-146a (rs2910164) to be associated with lower IS risk, but this trend was observed only in Koreans according to the allelic model. Our meta-analysis, in contrast, suggests that this $\mathrm{C}$ allele is not significantly associated with IS risk; instead, we found the GG genotype of miR-146a (rs2910164) to be associated with increased risk. Our result may be more reliable than that of the previous meta-analysis by $\mathrm{Zhu}$ et $\mathrm{al}^{39}$ because our meta-analysis contained nine more case-control studies ${ }^{14,15,17,21-26}$ with larger samples. Our subgroup analysis suggesting a significant relationship
A

\section{Study or subgroup}

Huang et al $(2015)^{20}$

Jeon et al $(2013)^{16}$

Liu et al $(2014)^{18}$

Lyu et al $(2016)^{23}$

Luo et al $(2017)^{25}$

Zhu et al $(2017)^{26}$

Zhu $(2016)^{24}$

Total $(95 \% \mathrm{CI})$

Total events

Heterogeneity: $\tau^{2}=0.02 ; \chi^{2}=12$

Test for overall effect: $Z=1.16(P=0.25)$
Allelic model (G-allele vs A-allele)

Weight OR M-H,

Events Total Events Total $(\%) \quad$ random, $95 \% \mathrm{Cl}$

$1.04(0.81-1.35)$

$0.94(0.77-1.16)$

$1.51(1.15-1.98)$

$0.91(0.70-1.18)$

$1.44(1.02-2.03)$

$0.97(0.78-1.20)$

$1.09(0.84-1.40)$

$1.09(0.94-1.25)$

$6,200 \quad 6,088 \quad 100 \quad 1.09(0.94-1.25)$

$0.04) ; I^{2}=54 \%$

) 
C

Study or subgroup

Huang et al $(2015)^{20}$

Jeon et al (2013) ${ }^{16}$

Liu et al $(2014)^{18}$

Lyu et al $(2016)^{23}$

Luo et al $(2017)^{25}$

Zhu et al $(2017)^{26}$

Zhu (2016) ${ }^{24}$

Total $(95 \% \mathrm{Cl})$

Total events

Heterogeneity: $\tau^{2}=0.04 ; \chi^{2}=16.43, d f=6(P=0.01) ; I^{2}=63 \%$

Test for overall effect: $Z=0.77(P=0.44)$

Dominant model (AA vs AG+GG)

Weight OR M-H,

Events Total Events Total $(\%)$ random, 95\% Cl

$\begin{array}{llllll}398 & 531 & 403 & 531 & 14.9 & 0.95(0.72-1.26)\end{array}$

$\begin{array}{llllll}460 & 678 & 365 & 553 & 16.4 & 1.09(0.86-1.38)\end{array}$

$\begin{array}{llllll}181 & 296 & 278 & 391 & 13.4 & 0.64(0.46-0.88)\end{array}$

$\begin{array}{llllll}257 & 378 & 250 & 378 & 14.0 & 1.09(0.80-1.47)\end{array}$

$\begin{array}{llllll}215 & 298 & 244 & 303 & 11.5 & 0.63(0.43-0.92)\end{array}$

$\begin{array}{llllll}349 & 505 & 328 & 510 & 15.5 & 1.24(0.96-1.61)\end{array}$

$\begin{array}{llllll}255 & 396 & 249 & 378 & 14.3 & 0.94(0.70-1.26)\end{array}$

$\begin{array}{rrrrr}3,115 & 3,082 & 3,044 & 100 & 0.93(0.78-1.12)\end{array}$

D

Study or subgroup

Huang et al (2015) ${ }^{20}$

Jeon et al $(2013)^{16}$

Liu et al $(2014)^{18}$

Lyu et al $(2016)^{23}$

Luo et al $(2017)^{25}$

Zhu et al $(2017)^{26}$

Zhu $(2016)^{24}$

Total $(95 \% \mathrm{Cl})$

Total events

Heterogeneity: $\chi^{2}=4.47, d f=5(P=0.48) ; l^{2}=0 \%$

Test for overall effect: $Z=1.25(P=0.21)$

\section{Homozygous model (GG vs AA)}

Weight OR M-H,

$\begin{array}{llllll}0 & 398 & 0 & 403 & \text { Not estimable }\end{array}$

$23 \quad 483 \quad 18 \quad 383 \quad 22.6 \quad 1.01(0.54-1.91)$

$\begin{array}{llllll}19 & 200 & 14 & 292 & 12.2 & 2.08(1.02-4.26)\end{array}$

$\begin{array}{llllll}11 & 268 & 15 & 265 & 17.1 & 0.71(0.32-1.58)\end{array}$

$\begin{array}{llllll}5 & 220 & 6 & 250 & 6.5 & 0.95(0.28-3.14)\end{array}$

$\begin{array}{llllll}32 & 381 & 24 & 352 & 27.0 & 1.25(0.72-2.17)\end{array}$

$\begin{array}{llllll}18 & 273 & 13 & 262 & 14.6 & 1.35(0.65-2.82)\end{array}$

$2,223 \quad 2,207 \quad 100 \quad 1.20(0.90-1.60)$
OR M-H,

random, $95 \% \mathrm{Cl}$

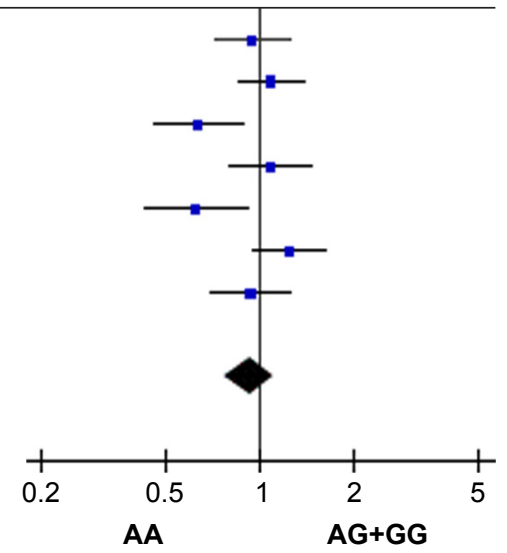


between the C allele of miR-146a (rs2910164) and lower IS risk contained only one case-control study, which was by Jeon et al. ${ }^{16}$

While the meta-analysis by $\mathrm{Zhu}$ et $\mathrm{al}^{39}$ reported an association between the A allele of miR-499 (rs3746444) and decreased IS risk in Chinese, our meta-analysis did
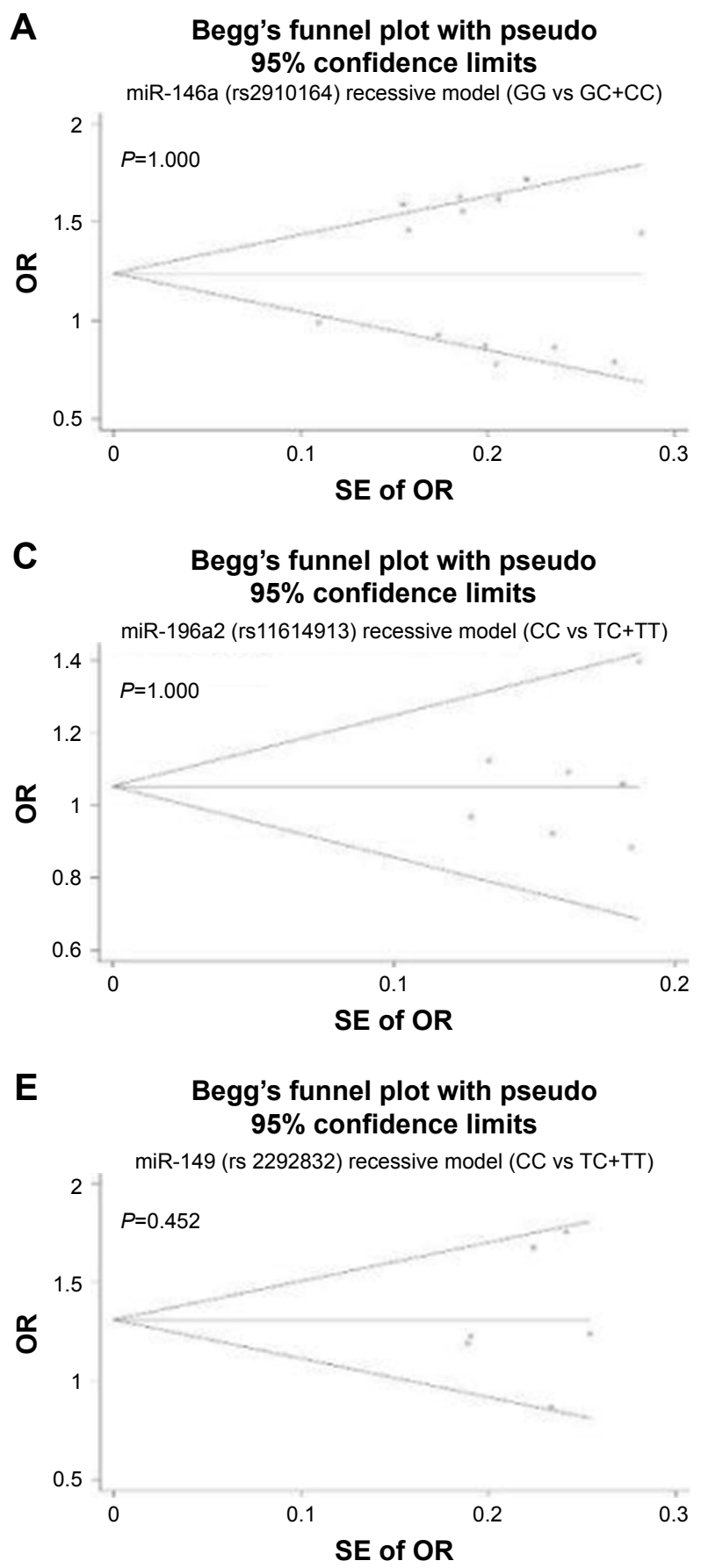

Figure 6 (Continued) not detect this association, either across Asian populations or specifically in the Chinese population (data not shown). Our result may be more reliable because our meta-analysis included four more case-control studies ${ }^{23-26}$ than the one by Zhu et al. ${ }^{39}$ The results of our meta-analysis are consistent with those reported in the meta-analysis by Xiao et al. ${ }^{37}$

B

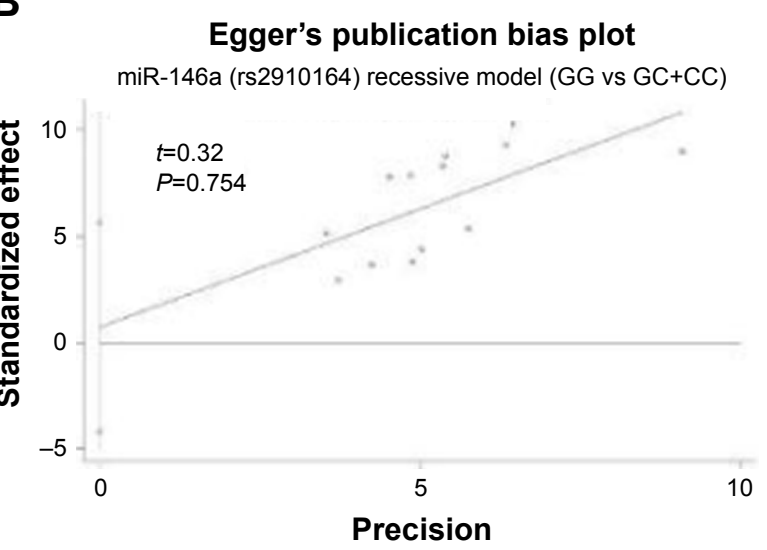

$\mathbf{D}$

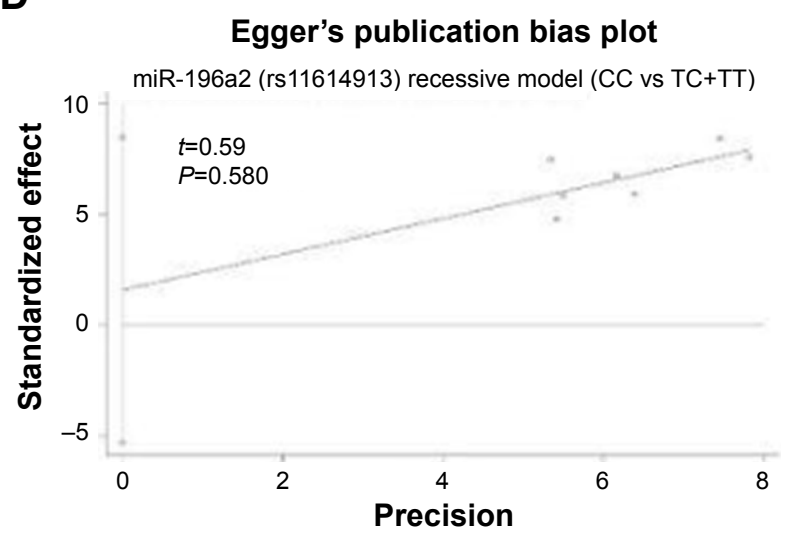

$\mathbf{F}$

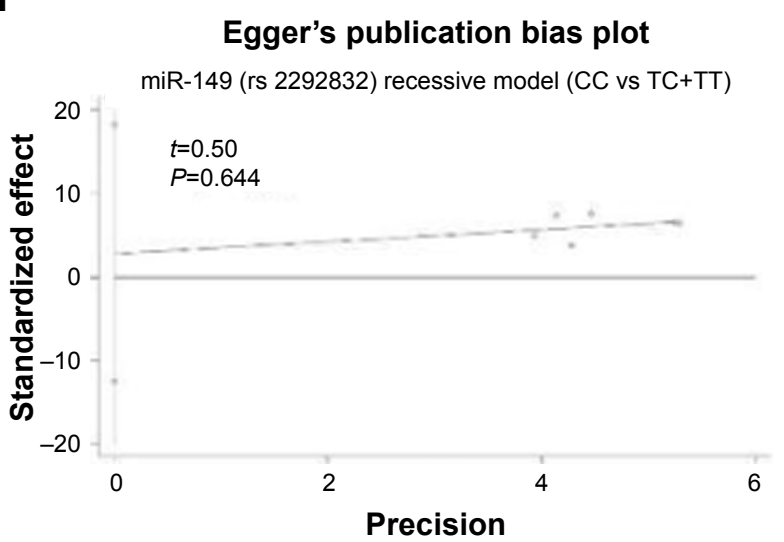



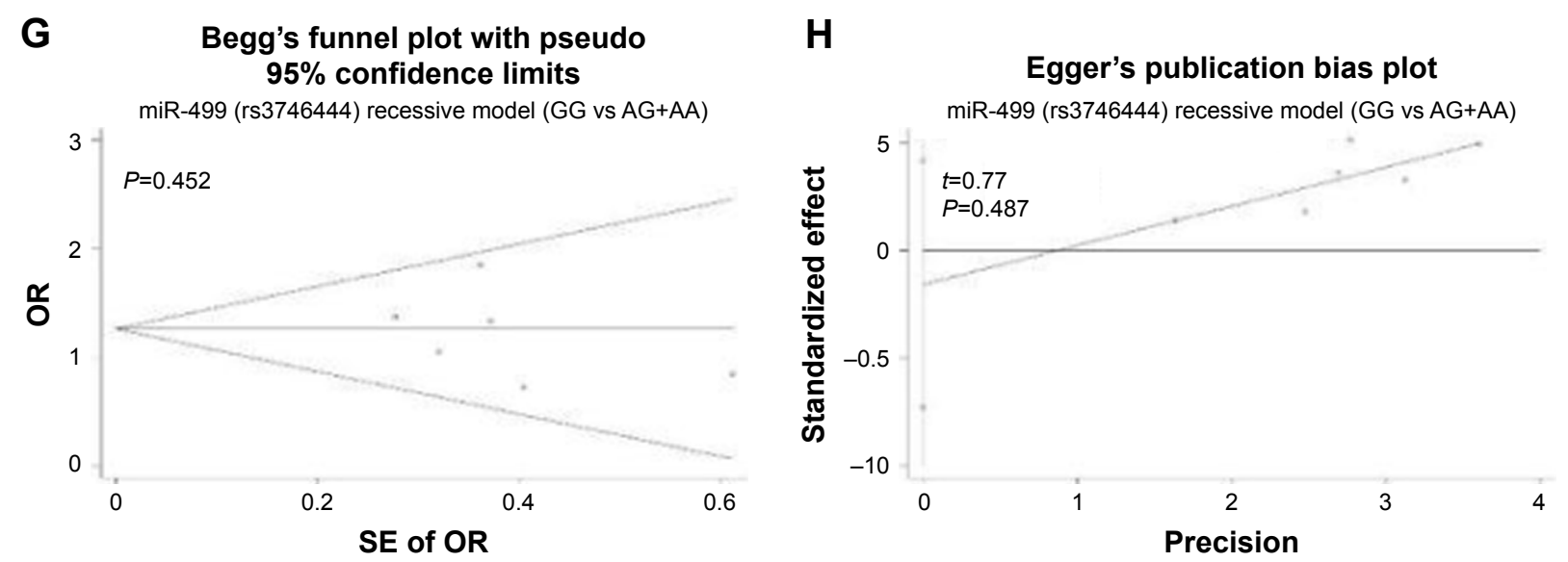

Figure 6 Begg's funnel plot and Egger's test to assess publication bias in the meta-analysis of potential associations between ischemic stroke risk and (A and B) miR-I46a (rs29l0164), (C and D) miR-196a2 (rs I 16I49I3), (E and F) miR-149 (rs2292832), and (G and H) miR-499 (rs3746444).

Note: All analyses were performed using a recessive genetic model.

Our meta-analysis suggests a significant association between the CC genotype of miR-149 (rs2292832) and increased IS risk. In contrast, the meta-analysis of Xiao et al ${ }^{37}$ based on two case-control studies indicated that the TT genotype and T allele of miR-149 (rs2292832) are associated with significantly lower IS risk, whereas another meta-analysis ${ }^{36}$ based on three case-control studies found the CC genotype and C allele of miR-149 (rs2292832) to be significantly associated with IS risk. Our meta-analysis contained three more case-control studies ${ }^{24-26}$ than either of these other metaanalyses, which may make it more reliable.

Our meta-analysis did not find a significant association between miR-196a2 (rs11614913) polymorphism and IS risk. This result confirms other meta-analyses ${ }^{37-39}$ based on smaller samples.

To the best of our knowledge, the current meta-analysis involves the largest sample (6,083 cases and 7,248 controls) than previous studies ${ }^{36-39}$ investigating the possible association of IS risk with miR-146a (rs2910164), miR196a2 (rs11614913), miR-149 (rs2292832), and miR-499 (rs3746444) in Asian populations. Nevertheless, the metaanalysis is limited by the designs of the included studies. First, the $P$-value for HWE was less than 0.05 in two studies $^{14,22}$ on miR-146a (rs2910164) and one study ${ }^{26}$ on miR-499 (rs3746444). These results suggested that these study populations may not be representative of the broader target population. Second, the results may be affected by both genetic and environmental factors, but most studies did not report environmental exposure, making it impossible to include them in the meta-analysis. Third, our exclusion of unpublished data and of papers published in languages other than English and Chinese may have biased our results. Fourth, the studies may be subject to performance bias, attrition bias, and reporting bias, although Newcastle-Ottawa scores were at least 5 for all 14 studies, indicating high quality. Fifth, stroke is a heterogeneous disease and has different subtypes that may affect the results of genetic association studies, but most case-control studies in our meta-analysis appeared not to use a well-phenotyped population. This may make the results less accurate. Finally, all the patients in this meta-analysis were Asian and this may limit the relevance of the results to other populations. Thus, more large and welldesigned studies are warranted in non-Asian populations.

\section{Conclusion}

This meta-analysis suggests that the GG genotype of miR-146a (rs2910164) and the CC genotype of miR-149 (rs2292832) may confer increased susceptibility to IS in Asian populations, whereas polymorphism in miR-196a2 (rs11614913) and miR-499 (rs3746444) may not be associated with IS risk. These conclusions should be verified in large and well-designed studies.

\section{Acknowledgments}

This study was supported by grants from the Natural Science Foundation of China (81560205 and 81760217), the Guangxi Natural Science Foundation (2016GXNSFCA380012 and 2017GXNSFAA198135), the Guangxi Colleges and Universities Science and Technology Research Project (KY2015ZD030), the Project of Nanning Scientific Research and Technology Development Plan (20163142), and the Scientific Research Project of Guangxi Health and Family Planning Commission (Z20170001) and China Scholarship Council (201708455059).

\section{Author contributions}

The study was designed by JRW and LC. The research was performed by DHZ, CBL, and QZ. Statistical analyses were 
performed by XFL, GQ, QH, and YSM. The manuscript was written by DHZ. All authors contributed toward data analysis, drafting and critically revising the paper and agree to be accountable for all aspects of the work.

\section{Disclosure}

The authors report no conflicts of interest in this work.

\section{References}

1. Roger VL, Go AS, Lloyd-Jones DM, et al. Heart disease and stroke statistics - 2012 update: a report from the American Heart Association. Circulation. 2012;125(1):e2-e220.

2. Lo AC, Guarino P, Krebs HI, et al. Multicenter randomized trial of robot-assisted rehabilitation for chronic stroke: methods and entry characteristics for VA ROBOTICS. Neurorehabil Neural Repair. 2009; 23(8):775-783.

3. Norrving B, Kissela B. The global burden of stroke and need for a continuum of care. Neurology. 2013;80(3 Suppl 2):S5-S12.

4. Dichgans M. Genetics of ischaemic stroke. Lancet Neurol. 2007; 6(2):149-161.

5. Holliday EG, Maguire JM, Evans TJ, et al. Common variants at 6p21.1 are associated with large artery atherosclerotic stroke. Nat Genet. 2012; 44(10):1147-1151.

6. International Stroke Genetics Consortium (ISGC), Wellcome Trust Case Control Consortium 2 (WTCCC2), Bellenguez C, Bevan S, Gschwendtner A, et al. Genome-wide association study identifies a variant in HDAC9 associated with large vessel ischemic stroke. Nat Genet. 2012;44(3):328-333.

7. Hachiya T, Kamatani Y, Takahashi A, et al. Genetic predisposition to ischemic stroke. Stroke. 2017;48(2):253-258.

8. Malik R, Chauhan G, Traylor M, et al. Multiancestry genome-wide association study of 520,000 subjects identifies 32 loci associated with stroke and stroke subtypes. Nat Genet. 2018;50(4):524-537.

9. Lewis BP, Burge CB, Bartel DP. Conserved seed pairing, often flanked by adenosines, indicates that thousands of human genes are microRNA targets. Cell. 2005;120(1):15-20.

10. Rink C, Khanna S. MicroRNA in ischemic stroke etiology and pathology. Physiol Genomics. 2011;43(10):521-528.

11. Ambros V. The functions of animal microRNAs. Nature. 2004;431(7006): 350-355.

12. Tan KS, Armugam A, Sepramaniam S, et al. Expression profile of MicroRNAs in young stroke patients. PLoS One. 2009;4(11):e7689.

13. Sun J. Association of miRNA-146a and EPHX2 Polymorphisms with Risk of Ischemic Stroke in Changsha Han Population and the Mechanisms [master's thesis]. Changsha, Hunan Province, China: Central South University; 2011.

14. Li L. Association of miRNA-146a Polymorphism with Risk of Cardiovascular Disease and Ischemia Stroke and the Mechanisms [master's thesis]. Changsha, Hunan Province, China: Central South University; 2010.

15. He S, Han Y. Association between miR-149 polymorphism and ischemic stroke of Han population in Hanzhong of Shanxi. J Mod Lab Med. 2013;2834:3238

16. Jeon YJ, Kim OJ, Kim SY, et al. Association of the miR-146a, miR-149, miR-196a2, and miR-499 polymorphisms with ischemic stroke and silent brain infarction risk. Arterioscler Thromb Vasc Biol. 2013;33(2):420-430.

17. Hu Y, Li S, Jiang X, et al. Study on the association of miR-146aC $>$ G, miR-149 T > C polymorphism with susceptibility to ischemic stroke. Prog Mod Biomed. 2014;14:5648-5651.

18. Liu Y, Ma Y, Zhang B, Wang SX, Wang XM, Yu JM. Genetic polymorphisms in pre-microRNAs and risk of ischemic stroke in a Chinese population. J Mol Neurosci. 2014;52(4):473-480.

19. Zhu R, Liu X, He Z, Li Q. miR-146a and miR-196a2 polymorphisms in patients with ischemic stroke in the northern Chinese Han population. Neurochem Res. 2014;39(9):1709-1716.
20. Huang S, Zhou S, Zhang Y, et al. Association of the genetic polymorphisms in pre-microRNAs with risk of ischemic stroke in a Chinese population. PLoS One. 2015;10(2):e0117007.

21. Zhong H, Cai Y, Cheng J, et al. Apolipoprotein E Epsilon 4 enhances the association between the rs2910164 polymorphism of mir-146a and risk of atherosclerotic cerebral infarction. $J$ Atheroscler Thromb. 2016;23(7):819-829.

22. Qu JY, Xi J, Zhang YH, et al. Association of the microRNA-146a SNP rs2910164 with ischemic stroke incidence and prognosis in a chinese population. Int J Mol Sci. 2016;17(5):660.

23. Lyu G, Wang S, Wang Q. Association of miR-146a rs2910164 and miR-499 rs3746444 polymorphisms with risk of ischemic stroke. New Med. 2016;47:257.

24. Zhu X. Association of miRNAs and MTHFR Gene Polymorphisms with Ischemic Stroke in the Chinese Han Population [Ph.D. thesis]. Qingdao, Shandong Province, China: Qingdao University; 2016.

25. Luo HC, Luo QS, Wang CF, Lei M, Li BL, Wei YS. Association of miR-146a, miR-149, miR-196a2, miR-499 gene polymorphisms with ischemic stroke in a Chinese people. Oncotarget. 2017;8(46):81295.

26. Zhu H, Zhang H, Bao L, Dai M. Analysis of association of genetic polymorphisms of microRNAs with ischemic stroke. Chin J Med Genet. 2017;34:261-265.

27. Wells GA, Shea B, O'connell D, et al. Newcastle-Ottawa Scale (NOS) for Assessing the Quality of NonranStudies in Meta-analyses. Available from: http://www.ohri.ca/programs/clinical_epidemiology/oxford.htm. Available in March, 2016

28. Ownby RL, Crocco E, Acevedo A, John V, Loewenstein D. Depression and risk for Alzheimer disease: systematic review, meta-analysis, and metaregression analysis. Arch Gen Psychiatry. 2006;63(5):530-538.

29. Shen J, Ambrosone CB, Dicioccio RA, Odunsi K, Lele SB, Zhao H. A functional polymorphism in the miR-146a gene and age of familial breast/ovarian cancer diagnosis. Carcinogenesis. 2008;29(10): 1963-1966.

30. Hoffman AE, Zheng T, Yi C, et al. microRNA miR-196a-2 and breast cancer: a genetic and epigenetic association study and functional analysis. Cancer Res. 2009;69(14):5970-5977.

31. El Gazzar M, Church A, Liu T, Mccall CE. MicroRNA-146a regulates both transcription silencing and translation disruption of TNF- $\alpha$ during TLR4-induced gene reprogramming.JLeukoc Biol. 2011;90(3):509-519.

32. Wu C, Gong Y, Sun A, et al. The human MTHFR rs4846049 polymorphism increases coronary heart disease risk through modifying miRNA binding. Nutr Metab Cardiovasc Dis. 2013;23(7):693-698.

33. Luthra R, Singh RR, Luthra MG, et al. MicroRNA-196a targets annexin A1: a microRNA-mediated mechanism of annexin A1 downregulation in cancers. Oncogene. 2008;27(52):6667-6678.

34. Yang B, Chen J, Li Y, et al. Association of polymorphisms in premiRNA with inflammatory biomarkers in rheumatoid arthritis in the Chinese Han population. Hum Immunol. 2012;73(1):101-106.

35. Cheng HS, Sivachandran N, Lau A, et al. MicroRNA-146 represses endothelial activation by inhibiting pro-inflammatory pathways. EMBO Mol Med. 2013;5(7):1017-1034.

36. du J, Cui C, Zhang S, Yang X, Lou J. Association of microRNA-146a and microRNA-149 polymorphisms with strokes in asian populations: an updated meta-analysis. Angiology. 2017;68(10):863-870.

37. Xiao Y, Bao MH, Luo HQ, Xiang J, Li JM. A Meta-analysis of the association between polymorphisms in microRNAs and risk of ischemic stroke. Genes. 2015;6(4):1283-1299.

38. Qin B, Zheng Y, Zhang W, et al. Lack of associations between rs2910164 and rs11614913 polymorphisms and the risk of ischemic stroke. Int J Clin Exp Med. 2015;8(10):18359-18366.

39. Zhu J, Yue H, Qiao C, Li Y. Association between single-nucleotide polymorphism (SNP) in miR-146a, miR-196a2, and miR-499 and risk of ischemic stroke: a meta-analysis. Med Sci Monit. 2015;21:3658-3663. 


\section{Publish your work in this journal}

Clinical Interventions in Aging is an international, peer-reviewed journal focusing on evidence-based reports on the value or lack thereof of treatments intended to prevent or delay the onset of maladaptive correlates of aging in human beings. This journal is indexed on PubMed Central, MedLine,

CAS, Scopus and the Elsevier Bibliographic databases. The manuscript management system is completely online and includes a very quick and fair peer-review system, which is all easy to use. Visit http://www.dovepress. com/testimonials.php to read real quotes from published authors. 